\title{
HIDROMEDUSAS DEL PACÍFICO COLOMBIANO: ASPECTOS BÁSICOS DE SU DINÁMICA ECOLÓGICA
}

\section{HYDROMEDUSAE OF THE COLOMBIAN PACÍFIC: BASIC ASPECTS OF THEIR ECOLOGICAL DYNAMICS}

Angela María Baldrich Chaparro • Raúl Hernando López Peralta²,3

\section{RESÚMEN}

Este estudio examina la dinámica espacio-temporal de la comunidad de hidromedusas en el Océano Pacífico Colombiano (OPC) en relación con la temperatura, salinidad, biomasa mesozooplanctónica y variación día/noche en la superficie, usando algunos análisis estadísticos e índices ecológicos. Un total de 128 muestras de mesozooplancton fueron obtenidas mediante arrastres superficiales con una red cónica $(50 \mathrm{~cm}$ de diámetro, malla de $363 \mu \mathrm{m}$ provista de un flujómetro para estimar el volumen de agua filtrada) durante cinco cruceros (23 junio-12 julio 2001, 27 agosto-15 septiembre 2001, 03-22 septiembre 2002, 01-21 septiembre 2003 y 18 septiembre-08 octubre de 2004). Las densidades más altas se presentaron durante septiembre de 2002 y septiembre-octubre de 2004, quizás principalmente por la mayor oferta de alimento. Se identificaron nueve especies meroplanctónicas y nueve holoplanctónicas, cuyos ciclos de vida aún son desconocidos o poco comprendidos; las especies holoplanctónicas dominantes Aglaura hemistoma, Liriope tetraphylla y Cytaeis tetrastyla fueron definidas como euritípicas en el OPC. La diversidad más alta $\left(H^{\prime}=1,65\right)$ fue menor que en otras regiones de Pacífico americano. No se observó una secuencia clara en la aparición de especies exclusivas, características o generalistas, ni un patrón de distribución espacio-temporal, aunque si indicios de migración nocturna a aguas superficiales. La similaridad entre las estaciones se explicó por las variables abióticas o bióticas, más que por su ubicación costera, intermedia u oceánica, o por la hora de los registros. Esta aproximación ecológica preliminar refleja la complejidad ambiental del OPC y el escaso conocimiento del comportamiento de la comunidad de las hidromedusas en el OPC.

Palabras clave: hidromedusas, diversidad, variación espacio-temporal, Pacífico colombiano, ERFEN.

1. Angela María Baldrich Chaparro, Cand. M. Sc. Producción, Manejo y Conservación de Recursos Naturales, U. de los Lagos, Camino Chinquihue km 6 Puerto Montt, Chile.

2. Raúl Hernando López Peralta, Dr. rer. nat. Laboratorio de Hidrobiología, Facultad de Ciencias Básicas y Aplicadas, Programa de Biología Aplicada, Campus Nueva Granada, U. Militar Nueva Granada, km 2 vía Cajicá-Zipaquirá, Colombia.

3. Autor para correspondencia: raul.lopez@unimilitar.edu.co. 


\section{ABSTRACT}

This study examines the spatio-temporal dynamics of hydromdeusae in the Colombian Pacific Ocean (OPC) in relation to surface temperature and salinity, mesozooplankton biomass and day/night variations, using some statistical analysis and ecological indexes. 128 mesozooplankton samples were obtained by means of surface tows, with a conical net $(50 \mathrm{~cm}$ diameter, $363 \mu \mathrm{m}$ mesh with a flowmeter to estimate the volume of water filtered) during five cruises (23 June-12 July 2002, 27 August-15 September 2001, 03-22 September 2002, 01-21 September 2003, 18 September-08 October 2004). The highest densities occurred during September 2002 and September-October 04, perhaps mainly due to the food availability increase. Nine holoplanktonic and nine meroplanktonic species were identified, whose life cycles are unknown or poorly understood; the dominant holoplanktonic species Aglaura hemistoma, Liriope tetraphylla and Cytaeis tetrastyla were defined as eurytopic in the OPC. The highest diversity $\left(H^{\prime}=1,65\right)$ was lower than in other regions of the American Pacific. No clear sequence in the occurrence of exclusive, characteristics or generalist species, or a pattern of spatial-temporal distribution were observed, but signs of night migration to surface waters. Similarity among stations was explained by abiotic or biotic variables, rather than their coastal, intermediate or oceanic location, or due to the records-time. It is estimated that this preliminary ecological approach reflects the environmental complexity of the OPC and the scarce knowledge of the behavior in the hydromedusae community in the OPC.

Key words: hidromedusae, diversity, spatio-temporal variation, colombian Pacific, ERFEN.

\section{INTRODUCCIÓN}

Las hidromedusas son representantes gelatinosos del zooplancton de ambientes marinos y estuarinos, donde generan un impacto considerable en la estructura y dinámica de esa comunidad por su gran actividad depredadora, a veces con especial énfasis sobre huevos y larvas de peces (ictioplancton), por lo que son de importancia en las evaluaciones pesqueras. También consumen copépodos, eufáusidos, quetognatos (éstos últimos pudiendo llegar a triplicarlas en tamaño), anfípodos gamáridos y, ocasionalmente, otras hidromedusas, siendo el canibalismo un evento frecuente dentro del grupo. A su vez, son alimento de diferentes especies de peces, tortugas y otros vertebrados marinos (Alvariño 1985, Hansson et al. 2005, Hansson y Kiørboe 2006, Møller y Riisgård 2007, Prudkovsky 2013).
Estos organismos tienen especies holoplanctónicas y meroplanctónicas, que pueden caracterizarse por: 1. El desarrollo directo de la larva plánula en una medusa. 2. Una fase de pólipo sésil y otra de medusa planctónica. 3. Ciclos de vida complejos endocelulares parasitarios (Bouillon y Boero 2000, Daly et al., 2007). Su distribución está influenciada por estas características, así como por sus migraciones verticales circadianas y factores abióticos como la salinidad, la temperatura y el régimen de corrientes, que afectan positiva o negativamente la densidad y diversidad de sus poblaciones. Algunas especies son indicadoras de cambios en las variables oceanográficas y masas de agua (Ramírez y Zamponi 1981, Benović et al., 2000, 2005, Suárez et al., 2002). Las hidromedusas pueden conformar "blooms" o proliferaciones 
masivas súbitas mono-específicas en zonas de productividad alta y en aguas someras, relacionados con una tasa reproductiva elevada e intensa, y son diferentes de las agregaciones causadas por los procesos de advección o concentración. Estos eventos pueden ocasionar un impacto negativo considerable sobre otros zoopláncteres (CIESM 2001, Graham et al., 2001, Mills 2001, Miglietta et al., 2008).

Por todas las razones expuestas, el estudio de estos organismos permite un mayor entendimiento del funcionamiento ecológico de los ecosistemas donde habitan (Palma y Rosales 1995, Suárez et al. 1999).

En el Pacífico Oriental se han realizado estudios sobre su abundancia y distribución geográfica, estacional, batimétrica y nictimeral, e. g., en aguas de California y Baja California (Alvariño 1999), México (Segura et al.,2003, 2010), bahía Culebra, golfo de Papagayo (Costa Rica) (Rodríguez et al., 2012), bahía de Panamá (Miglietta et al., 2008), Bahía de Santa Elena (Ecuador) (Andrade 2012) y sobre todo a lo largo de Chile (Palma y Rosales 1995, Apablaza y Palma, 2003, 2006, Palma y Apablaza 2004, Palma et al., 2007, Villenas et al., 2009, Pavez et al., 2010, Bravo et al., 2011, Mattos y Mujica 2012). Es de resaltar el de Segura (1984), que incluye morfología, sistemática y zoogeografía de especies del Pacífico Tropical Oriental (PTO).

En el Océano Pacífico Colombiano (OPC) los estudios de hidromedusas son aún incipientes. Alvariño (1978) y Segura (1984) referenciaron la distribución y abundancia de sólo algunas especies en el OPC. En aguas costeras Cely y Chiquillo (1993) evaluaron su distribución e identificaron algunas especies. López (1997), las incluyó dentro de su estudio del ictioplancton pero sin identificarlas. Recientemente, se elaboró el primer listado de géneros y especies (López y Baldrich 2010) y el primer catálogo de identificación de hidromedusas (Baldrich y López 2010) del área de estudio.

Este trabajo se enmarca dentro de los estudios de la dinámica ecológica del zooplancton durante los cruceros de la serie ERFEN (Estudio Regional del Fenómeno El Niño) en el OPC, llevados a cabo por el Grupo de Investigación en Hidrobiología Aplicada (HIDROBIA) de la Universidad Militar Nueva Granada (UMNG). Los objetivos del trabajo incluyeron la identificación taxonómica de las hidromedusas y la evaluación de su distribución y abundancia en relación con la temperatura, salinidad, biomasa mesozooplanctónica y variación día/noche en la superficie.

La hipótesis es que la distribución de las hidromedusas en el OPC está mediada por la disponibilidad de alimento, la temperatura y, en especial la salinidad debido a la influencia dulceacuícola en la zona costera, así como por la migración a la superficie en horas de la noche. Es de esperarse la presencia de espcies mero- y holoplanctónicas, comunes en los arrastres de zooplancton en otras regiones del Pacífico americano.

\section{MATERIALES Y MÉTODOS}

\section{Área de estudio}

El área de estudio corresponde al OPC (ca. $349.000 \mathrm{~km}^{2}$ ), comprendido entre los 01 ${ }^{\circ} 30^{\prime}$ y $6^{\circ} 30^{\prime}$ de latitud norte y entre los $77^{\circ} 45^{\prime}$ y $84^{\circ} 00^{\prime}$ de longitud oeste. Al sur bordea la frontera con Ecuador, al norte la frontera con Panamá y al oriente está limitada por 1.300 km de costa (CCCP 2002) (Fig. 1). Hace parte de la región conocida como Panama Bight, ubicada dentro de la Concavidad Ecuatorial de Baja Presión, en la cual se encuentra la Zona de Convergencia Intertropical (ZCIT) (CCCP 2002).

Con base en varios autores (e. g., Forsbergh 1963, Wirtky 1965, Stevenson et al., 1970, Tchantsev y Cabrera 1998, CCCP 2002, Villegas 2002, 2003, Devis 2003, Rodríguez et al., 2003), los atributos más relevantes del área de estudio son: 1. Por su ubicación en la ZCIT está influenciada por corrientes de viento variables, masas de nubes y precipitaciones fuertes; de hecho, el área de estudio es considerada una de las más lluviosas del 
mundo, con un promedio acumulado de precipitación costera de 17.057 mm/año. 2. Cuenta con numerosos ríos, muchos caudalosos, que transportan una carga sedimentaria alta. 3. Su topografía dinámica superficial es muy compleja, pues tiene la influencia de las corrientes: Ecuatorial del Norte, Contracorriente Ecuatorial y del Golfo de Panamá, y en su cuenca se forman la corriente de Colombia, que acarrea aguas oceánicas de baja salinidad y temperatura hacia la costa, y la contrarriente del Chocó, que arrastra aguas de la ensenada de Panamá. A lo anterior se suman los procesos temporales de surgencia oceánica y costera durante casi todo el año en grandes extensiones, al igual que

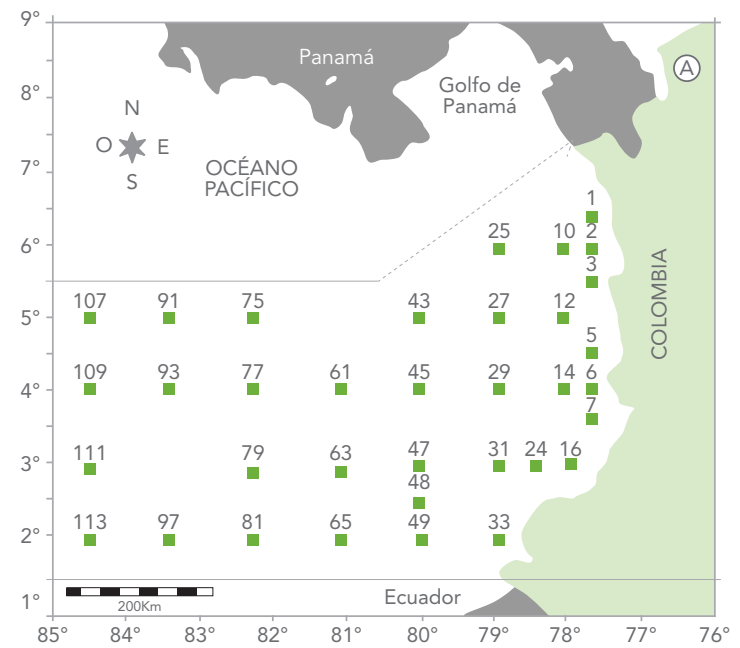

efectos de cabo (e. g. Cabo Corrientes) e isla (Gorgona y Malpelo), y remolinos de alta productividad planctónica, además de un amplio rango de mareas de máximo $6 \mathrm{~m}$. 4. Es afectada frecuentemente por episodios El Niño y la Niña de diferente duración e intensidad. 5. Cuando no ocurren estos eventos, i. e., condiciones normales, la temperatura superficial varía entre 26,4 y $28,0^{\circ} \mathrm{C}$, y la salinidad entre $30,0-34,9$, con una diferencia marcada entre las aguas costeras y las oceánicas. Los máximos de salinidad se observan de febrero a marzo y los mínimos en diciembre; la clorofila a varía entre $0-4,6 \mathrm{mg} / \mathrm{m}^{3}$.

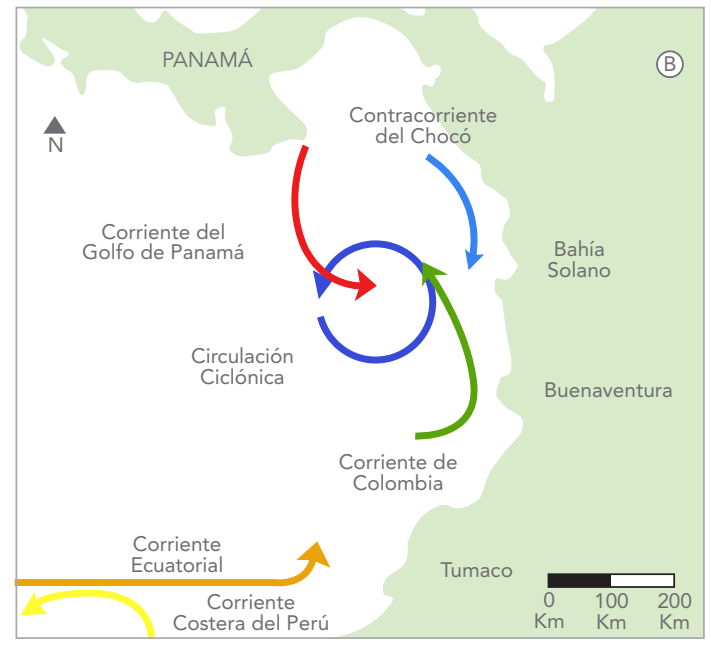

Fig. 1. A. Estaciones ERFEN para la captura de zooplancton y estudio de las hidromedusas. B. principales corrientes superficiales en el OPC. - Estaciones de estudio de la comunidad de hidromedusas-mesozooplancton.

Actividades durante las campañas oceanográficas y en laboratorio

En las aguas del OPC, el Centro Control de Contaminación del Pacífico (CCCP) estableció un total de 113 estaciones separadas por 30 millas náuticas (mn) en las aguas costeras y por $60 \mathrm{mn}$ en las intermedias y oceánicas, diferenciando entre estaciones biológicas (captura de zooplancton) y no biológicas (determinación exclusiva de variables físico-químicas). Este estudio se desarrolló en la época hidrográfica cálida (mayo-diciembre), definida por la ubicación $\approx 10^{\circ} \mathrm{N}$ de la ZCIT (Rodríguez et al., 2003, Devis et al.,2008). Se seleccionaron cinco cruceros oceanográficos con diferente número de estaciones (en paréntesis): 23 de junio-12 de julio de 2001 (30), 27 de agosto-15 de septiembre de 2001 (23), 03-22 de septiembre de 2002 (25), 01-21 de septiembre de 2003 (25) y 18 de septiembre-08 de octubre de 2004 (25) (Tabla 1). En el texto se abreviaron como: Jun-Jul.01, Ago-Sep.01, Sep.02, Sep.03 y Sep-Oct.04. 


\begin{tabular}{|c|c|c|c|c|c|c|c|c|c|c|c|c|}
\hline \multirow{2}{*}{ Estación } & \multicolumn{2}{|c|}{ Coordenadas } & \multicolumn{2}{|c|}{ Jun-Jul.01 } & \multicolumn{2}{|c|}{ Ago-Sep.01 } & \multicolumn{2}{|c|}{ Sep.02 } & \multicolumn{2}{|c|}{ Sep.03 } & \multicolumn{2}{|c|}{ Sep-Oct.04 } \\
\hline & $\begin{array}{l}\text { Latitud } \\
\text { Norte }\end{array}$ & $\begin{array}{c}\text { Longitud } \\
\text { Oeste }\end{array}$ & Fecha & Hora & Fecha & Hora & Fecha & Hora & Fecha & Hora & Hora & Fecha \\
\hline 1 & $06^{\circ} 05^{\prime} 00^{\prime \prime}$ & $77^{\circ} 75^{\prime} 00^{\prime \prime}$ & & & & & 16.Sep & $19: 30$ & 06.Sep & $13: 10$ & 23.Sep & $04: 48$ \\
\hline 2 & $06^{\circ} 00^{\prime} 00^{\prime \prime}$ & $77^{\circ} 75^{\prime} 00^{\prime \prime}$ & 12.Jul & $18: 12$ & & & & & & & & \\
\hline 3 & $05^{\circ} 50^{\prime} 00^{\prime \prime}$ & $77^{\circ} 75^{\prime} 00^{\prime \prime}$ & 12.Jul & $20: 00$ & 14.Sep & $22: 30$ & 16.Sep & $10: 20$ & 05.Sep & $13: 55$ & 22.Sep & $20: 20$ \\
\hline 5 & $04^{\circ} 00^{\prime} 00^{\prime \prime}$ & $77^{\circ} 75^{\prime} 00^{\prime \prime}$ & & & 15.Sep & 07:00 & 16.Sep & $00: 30$ & 12.Sep & $13: 45$ & 21.Sep & $07: 45$ \\
\hline 6 & $04^{\circ} 00^{\prime} 00^{\prime \prime}$ & $77^{\circ} 75^{\prime} 00^{\prime \prime}$ & 08.Jul & 21:45 & & & & & & & & \\
\hline 7 & $03^{\circ} 50^{\prime} 00^{\prime \prime}$ & $77^{\circ} 75^{\prime} 00^{\prime \prime}$ & & & 06.Sep & 09:30 & 16.Sep & $17: 15$ & 02.Sep & $11: 24$ & 20.Sep & $02: 40$ \\
\hline 10 & $06^{\circ} 00^{\prime} 00^{\prime \prime}$ & $78^{\circ} 00^{\prime} 00^{\prime \prime}$ & & & 14.Sep & $18: 05$ & 16.Sep & $16: 00$ & 05.Sep & $18: 55$ & 23.Sep & $00: 13$ \\
\hline 12 & $05^{\circ} 00^{\prime} 00^{\prime \prime}$ & $78^{\circ} 00^{\prime} 00^{\prime \prime}$ & 10.Jul & $02: 56$ & 15.Sep & $02: 30$ & 16.Sep & $04: 40$ & 05.Sep & $00: 45$ & 22.Sep & $08: 15$ \\
\hline 14 & $04^{\circ} 00^{\prime} 00^{\prime \prime}$ & $78^{\circ} 00^{\prime} 00^{\prime \prime}$ & 09.Jul & $00: 10$ & 08.Sep & 05:00 & 14.Sep & $21: 30$ & 03.Sep & $19: 38$ & 21.Sep & $04: 15$ \\
\hline 16 & $03^{\circ} 00^{\prime} 00^{\prime \prime}$ & $78^{\circ} 00^{\prime} 00^{\prime \prime}$ & 05.Jul & $08: 50$ & 15.Sep & $23: 00$ & 22.Sep & $22: 15$ & 02.Sep & 07.05 & 19.Sep & $23: 15$ \\
\hline 24 & $03^{\circ} 00^{\prime} 00^{\prime \prime}$ & $78^{\circ} 50^{\prime} 00^{\prime \prime}$ & 05.Jul & $04: 00$ & & & & & & & & \\
\hline 25 & $06^{\circ} 00^{\prime} 00^{\prime \prime}$ & $79^{\circ} 00^{\prime} 00^{\prime \prime}$ & 10.Jul & 18:00 & 13.Sep & 08:00 & 17.Sep & 07:00 & 06.Sep & 02:12 & 25.Sep & $04: 45$ \\
\hline 27 & $05^{\circ} 00^{\prime} 00^{\prime \prime}$ & $79^{\circ} 00^{\prime} 00^{\prime \prime}$ & 03.Jul & $16: 30$ & 12.Sep & 22:35 & 18.Sep & $17: 30$ & 04.Sep & $17: 20$ & 22.Sep & 01:27 \\
\hline 29 & $04^{\circ} 00^{\prime} 00^{\prime \prime}$ & $79^{\circ} 00^{\prime} 00^{\prime \prime}$ & 04.Jul & 08:05 & 09.Sep & $12: 30$ & 22.Sep & 07:00 & 03.Sep & $12: 20$ & 20.Sep & $18: 50$ \\
\hline 31 & $03^{\circ} 00^{\prime} 00^{\prime \prime}$ & $79^{\circ} 00^{\prime} 00^{\prime \prime}$ & 05.Jul & $00: 05$ & 05.Sep & $00: 00$ & 09.Sep & $23: 00$ & 01.Sep & 23.45 & 19.Sep & $17: 00$ \\
\hline 33 & $02^{\circ} 00^{\prime} 00^{\prime \prime}$ & $79^{\circ} 00^{\prime} 00^{\prime \prime}$ & 23.Jun & $18: 40$ & 27.Ago & $12: 40$ & 03.Sep & 18:15 & 21.Sep & 12:03 & 08.Sep & 05.20 \\
\hline 43 & $05^{\circ} 00^{\prime} 00^{\prime \prime}$ & $80^{\circ} 00^{\prime} 00^{\prime \prime}$ & 03.Jul & 09:40 & & & 19.Sep & 01:30 & 09.Sep & 04:59 & 25.Sep & 20:15 \\
\hline 45 & $04^{\circ} 00^{\prime} 00^{\prime \prime}$ & $80^{\circ} 00^{\prime} 00^{\prime \prime}$ & 03.Jul & $02: 20$ & & & 21.Sep & $18: 30$ & 02.Sep & $05: 40$ & 28.Sep & $20: 30$ \\
\hline 47 & $03^{\circ} 00^{\prime} 00^{\prime \prime}$ & $80^{\circ} 00^{\prime} 00^{\prime \prime}$ & 02.Jul & $18: 30$ & 05.Sep & $14: 50$ & 09.Sep & 14:55 & 15.Sep & 06.18 & 07.Oct & $07: 55$ \\
\hline 48 & $02^{\circ} 50^{\prime} 00^{\prime \prime}$ & $80^{\circ} 00^{\prime} 00^{\prime \prime}$ & 24.Jun & 07:20 & & & & & & & & \\
\hline 49 & $02^{\circ} 00^{\prime} 00^{\prime \prime}$ & $80^{\circ} 00^{\prime} 00^{\prime \prime}$ & 24.Jun & $14: 30$ & 28.Ago & 01:00 & 04.Sep & $06: 00$ & 21.Sep & $18: 45$ & 04.Oct & $18: 30$ \\
\hline 61 & $04^{\circ} 00^{\prime} 00^{\prime \prime}$ & $81^{\circ} 00^{\prime} 00^{\prime \prime}$ & 02.Jul & 04:05 & 05.Sep & $00: 20$ & & & & & & \\
\hline 63 & $03^{\circ} 00^{\prime} 00^{\prime \prime}$ & $81^{\circ} 00^{\prime} 00^{\prime \prime}$ & 02.Jul & $11: 30$ & & & & & & & & \\
\hline 65 & $02^{\circ} 00^{\prime} 00^{\prime \prime}$ & $81^{\circ} 00^{\prime} 00^{\prime \prime}$ & 26.Jun & 01:30 & 29.Ago & 10:30 & & & & & & \\
\hline 75 & $05^{\circ} 00^{\prime} 00^{\prime \prime}$ & $82^{\circ} 00^{\prime} 00^{\prime \prime}$ & 30.Jun & $00: 00$ & 04.Sep & $11: 20$ & 19.Sep & 17:15 & 09.Sep & $23: 55$ & 28.Sep & $06: 45$ \\
\hline 77 & $04^{\circ} 00^{\prime} 00^{\prime \prime}$ & $82^{\circ} 00^{\prime} 00^{\prime \prime}$ & 29.Jun & $06: 25$ & 03.Sep & $00: 40$ & 21.Sep & $06: 00$ & 11.Sep & 12:18 & 26.Sep & $22: 30$ \\
\hline 79 & $03^{\circ} 00^{\prime} 00^{\prime \prime}$ & $82^{\circ} 00^{\prime} 00^{\prime \prime}$ & 28.Jun & $23: 00$ & 01.Sep & $09: 30$ & 09.Sep & $00: 38$ & 15.Sep & 20.34 & 04.Oct & $23: 30$ \\
\hline 81 & $02^{\circ} 00^{\prime} 00^{\prime \prime}$ & $82^{\circ} 00^{\prime} 00^{\prime \prime}$ & 26.Jun & 09:30 & & & 06.Sep & $01: 30$ & 21:Sep & 15:18 & 07.Oct & $02: 33$ \\
\hline 91 & $05^{\circ} 00^{\prime} 00^{\prime \prime}$ & $83^{\circ} 00^{\prime} 00^{\prime \prime}$ & 30.Jun & $17: 30$ & & & & & & & & \\
\hline 93 & $04^{\circ} 00^{\prime} 00^{\prime \prime}$ & $83^{\circ} 00^{\prime} 00^{\prime \prime}$ & 29.Jun & $16: 45$ & 03.Sep & 09:00 & & & & & & \\
\hline 97 & $02^{\circ} 00^{\prime} 00^{\prime \prime}$ & $83^{\circ} 00^{\prime} 00^{\prime \prime}$ & 27.Jun & 01:30 & 03.Sep & $03: 30$ & & & & & & \\
\hline 107 & $05^{\circ} 00^{\prime} 00^{\prime \prime}$ & $84^{\circ} 00^{\prime} 00^{\prime \prime}$ & 30.Jun & $10: 50$ & 01.Sep & $22: 20$ & 20.Sep & 09:10 & 10.Sep & $16: 10$ & 27.Sep & $18: 00$ \\
\hline 109 & $04^{\circ} 00^{\prime} 00^{\prime \prime}$ & $84^{\circ} 00^{\prime} 00^{\prime \prime}$ & 29.Jun & $16: 45$ & & & 20.Sep & 17:15 & 10.Sep & $23: 35$ & 27.Sep & $12: 30$ \\
\hline 111 & $03^{\circ} 00^{\prime} 00^{\prime \prime}$ & $84^{\circ} 00^{\prime} 00^{\prime \prime}$ & 27.Jun & 21:30 & 30.Ago & $23: 30$ & 07.Sep & 12:05 & 16.Sep & $10: 40$ & 06.Oct & $02: 00$ \\
\hline 113 & $02^{\circ} 00^{\prime} 00^{\prime \prime}$ & $84^{\circ} 00^{\prime} 00^{\prime \prime}$ & & & & & 07.Sep & $04: 00$ & 17.Sep & $18: 20$ & 06.Oct & $09: 15$ \\
\hline
\end{tabular}

Tabla 1. Ubicación de las estaciones de estudio de las hidromedusas-mesozooplancton en el OPC durante los cinco periodos de muestreo. 1-18 y 33 costeras, 26-49 intermedias, 76-113 oceánicas. En gris horas nocturnas. La interrupción de la secuencia en la numeración de las estaciones se explica porque no todas las estaciones fueron muestreadas. 
Los organismos fueron capturados en el primer metro superficial mediante arrastres circulares de 10 minutos empleando una red cónica de $50 \mathrm{~cm}$ de abertura y malla de $363 \mu \mathrm{m}$ de poro, lo que define individuos del mesozooplancton (Johnson y Allen 2005); a la boca de la red se fijó un flujómetro HydroBios para determinar el volumen de agua filtrada (Suthers y Rissik 2009). Las muestras se fijaron con formaldehído al $10 \%$ en agua de mar y se conservaron con formaldehído al 4\% (Raskoff et al., 2003).

La determinación de la temperatura y la salinidad se realizó con un perfilador contínuo CTD Sea Bird Electronics; sólo se consideraron los datos a $1 \mathrm{~m}$ de profundidad, designados como superficiales.

De las 128 muestras de mesozooplancton obtenidas en los cinco periodos se extrajeron en su totalidad las hidromedusas para su identificación. Las especies fueron identificadas siguiendo a Mayer (1910), Kramp (1961), Ramírez y Zamponi (1981), Segura (1984), Pagès et al., (1992) y Bouillon (1999).

\section{Análisis de la información}

Con el fin de conjugar unidades diferentes y ponderar por igual las variables abióticas con diferentes magnitudes, los valores de temperatura y salinidad en la superficie, se estandarizaron con la fórmula: $Z$ $=\mathrm{x}-\overline{\mathrm{x}} / \mathrm{S}(\mathrm{n}-1)$, donde Z: variable normal estandarizada, $\bar{x}$ : promedio muestreal, $x$ : valor de cada parámetro, $\mathrm{S}$ (n-1): desviación estándar (Ramírez 2005).

Las densidades de hidromedusas se estandarizaron con la fórmula: $\mathrm{N}=100 \mathrm{~m}^{3} \mathrm{n} / \pi \mathrm{r}^{2} \mathrm{~d}$ (Suthers y Rissik 2009), donde $\mathrm{N}$ es el número de hidromedusas/100 $\mathrm{m}^{3}$, n el número capturado en cada arrastre y $\pi r^{2} \mathrm{~d}$ el volumen de agua filtrada en cada caso; $r$ es el radio de la red y d el número de revoluciones por el factor de calibración del flujómetro $(0,3)$. Las densidades se transformaron a $\log (\mathrm{x}+1)$ para cumplir con los supuestos de normalidad y homogeneidad de varianzas y su variación cronológica general en los cinco periodos se comprobó con la prueba no paramétrica
Kruskal-Wallis ( $p=0,05$; significancia del 95\%) (Ramírez 2005, 2006).

Los datos de biomasa húmeda del mesozooplancton $\mathrm{g} / 100 \mathrm{~m}^{3}$, estandarizados igual que las densidades de hidromedusas, y las figuras correspondientes, fueron suministrados por el grupo HIDROBIA de la UMNG.

El análisis espacial se efectuó mediante la técnica UPGMA (Unweighted pair-group method with arithmetic mean= técnica de promedio no ponderado para pares de grupos) utilizando el índice de similitud Bray-Curtis y la ordenación NMDS (Non Metric Multidimensional Scaling= escalamiento multidimensional no métrico) (Ramírez 2005, 2006), contemplando las 16 estaciones comunes en los cinco periodos evaluados. Similitudes mayores al $60 \%$ se consideraron representativas de la unión de los grupos.

Con las asociaciones obtenidas se aplicó el análisis inverso de Kaandorp, que define los siguientes tipos de especies (Kaandorp 1986): 1. Exclusivas: encontradas en un solo grupo con el $100 \%$ de abundancia relativa. 2. Características: incluidas en más de un grupo y con más del $70 \%$ de abundancia relativa en uno en particular. 3. Generalistas: distribuidas de forma uniforme entre los grupos.

Con el fin de establecer la posible relación entre las densidades, la temperatura y la salinidad, se efectuó la prueba no paramétrica de Spearman $(p<0,05)$ (Zar 2010) (Hair et al., 1999), incluyendo la hora de captura (día= 06:00-18:00 h, noche= 18:01-05:59 h), para observar la probable influencia termo-halina sobre las densidades. Se utilizaron los programas Primer V5 ${ }^{\circledR}$, Statgraphics Plus $5^{\circledR}$ y Cluster para DOS.

Para el análisis de la estructura comunitaria por periodo se empleó el índice de uniformidad de Pielou (J') (Pielou 1966), J'= $\mathrm{H}^{\prime}{ }_{\text {obs }} / \mathrm{H}_{\text {máx' }}^{\prime}$ donde $\mathrm{H}^{\prime}$ : diversidad, calculado por medio de la expresión de Shannon-Weaver $H^{\prime}=-\left(n_{i} / N\right) \log _{2}\left(n_{i} / N\right)$, donde $n$ : número de individuos de la especie i, y $\mathrm{N}$ : número total de 
individuos (Shannon y Weaver 1949); el predominio de Simpson (1-Lambda) (Simpson 1949) y la riqueza, como número de especies (S) (Margalef 1958).

\section{RESULTADOS Y DISCUSIÓN}

Variables abióticas y variación espacio-temporal de las densidades generales de las hidromedusas

El registro de temperatura más alto $\left(27,50^{\circ} \mathrm{C}\right)$ fue en aguas neríticas en Sep.03 y el más bajo $\left(26,33^{\circ} \mathrm{C}\right)$ hacia la zona oceánica en Sep-Oct.04. La salinidad exhibió un gradiente horizontal inverso
(Fig.2), debido a las altas precipitaciones en la zona costera que aumentan los aportes fluviales (CCCP 2002), con promedios entre 30,93 (Sep-Oct.04) y 32,52 (Jun-Jul.01). En Sep.02 hubo un descenso evidente en la amplitud de los rangos salinos $(4,71)$ mientras en Sep-Oct.04 ocurrió lo contrario $(8,58)$, por el desplazamiento de la ZCIT que genera cambios en la frecuencia e intensidad de las precipitaciones (Fiedler y Talley 2006, Clarke 2008). Este cuadro termo-halino (Spearman=-0,824) correspondió a condiciones normales en los cinco periodos de estudio (CCCP, 2002).

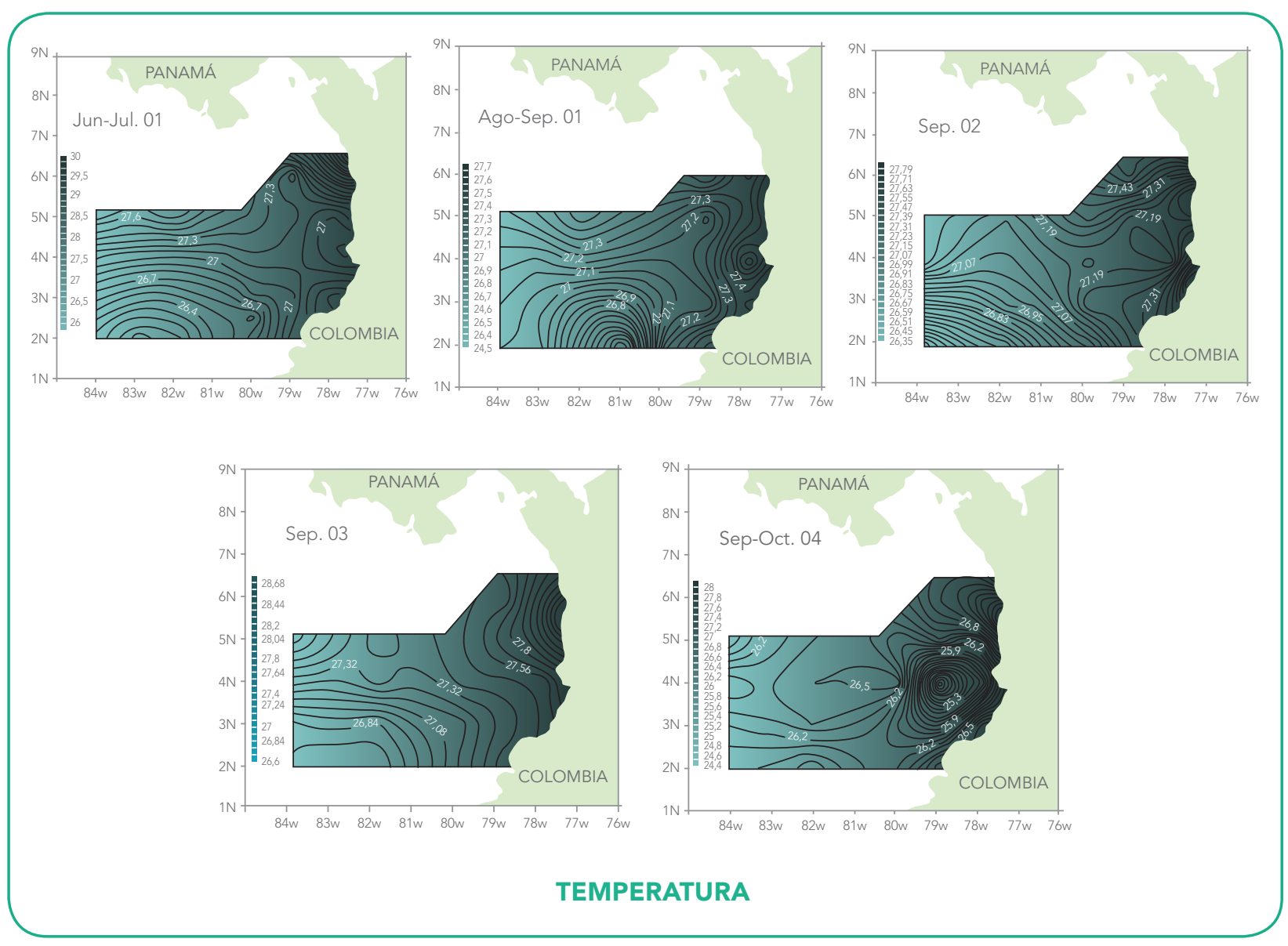



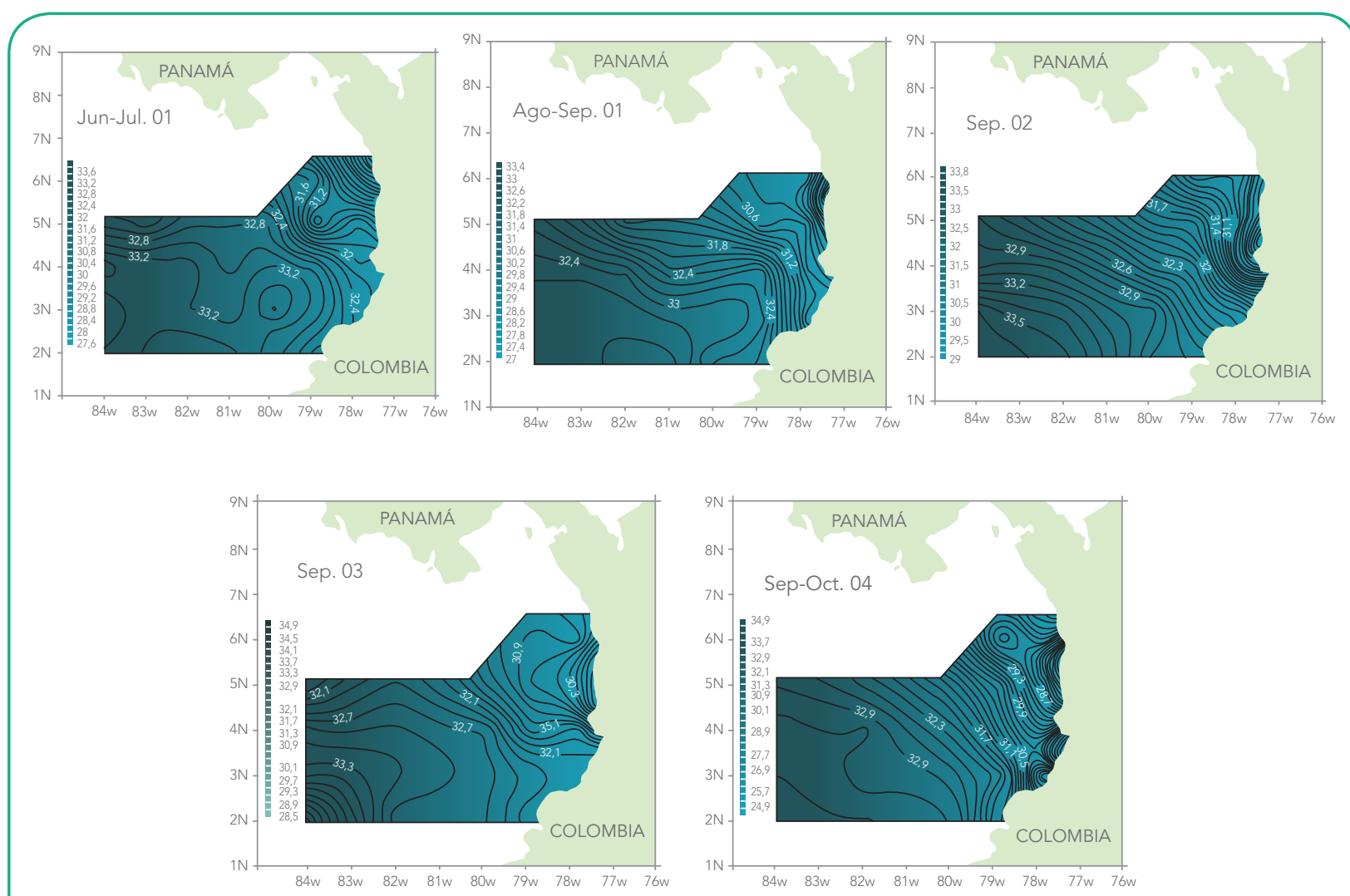

\section{SALINIDAD}

Fig. 2. Temperatura $\left({ }^{\circ} \mathrm{C}\right)$ y salinidad superficiales en el OPC durante los cinco periodos de estudio.

Las densidades de hidromedusas (\%) para cada periodo fueron: 8,09 (Jun-Jul.01), 11,02 (Ago-Sep.01), 13,44 (Sep.02), 9,51 (Sep.03) y 57,94 (Sep-Oct.04), cuya comparación estadística, incluyendo termperatura y salinidad, arrojó diferencias significativas (Kruskal-Wallis $=29,71 ; p=0,05$ ) entre los cinco periodos. Tanto en aguas costeras como oceánicas, las densidades fueron bajas ( $<15$ ind/100 $\mathrm{m}^{3}$ ) y medias (16-205 ind/100 $\left.\mathrm{m}^{3}\right)$, salvo en las estaciones costeras 33 y 49, donde alcanzaron 381 y 227 ind/100 m³, respectivamente, en Sep-Oct.04 (Fig. 3).

No se observaron patrones de dispersión espacial, posiblemente por la influencia de un conjunto de factores, como la migración vertical circadiana y los procesos hidrodinámicos que implican transporte, retención y dispersión de los pláncteres, en general, y otros que generan una alta productividad biológica y consecuente mayor disponibilidad de alimento para las hidromedusas y sus depredadores (e. g., surgencias, remolinos y frentes) (Harris et al., 2000, Mianzan et al., 2001, Prairie et al., 2012,. Prudkovsky 2013). Lo anterior se ha comprobado en otras regiones del Pacífico americano, e. g., Estados Unidos (Mackas y Galbraith 2002, Bi et al., 2011), Costa Rica (Morales y Brugnoli 2001, Morales 2008, Rodríguez et al., 2012), Panamá (Miglietta et al., 2008), Ecuador 
(Luzuriaga de Cruz 2009), Perú (Chávez et al., 2008) y Chile (Palma y Apablaza 2004, Apablaza y Palma 2006, Pavez et al., 2010, Bravo et al., 2011).

Las mayores concentraciones en algunos sectores se relacionarían, en parte, con la disponibilidad de alimento, pues las hidromedusas incluyen en su dieta un amplio espectro de zoopláncteres (Alvariño 1985, Vinogradov y Shushkina 2002, Benović et al., 2000, 2005, Hansson et al., 2005, Hansson y Kiørboe 2006, Møller y Riisgård 2007, Prudkovsky 2013). Las correlaciones positivas entre las hidromedusas y la biomasa de mesozooplancton en todos los periodos de estudio, en especial en Sep.02 y Sep.03 (Spearman 0,594 y 0,519 respectivamente), sugieren esta particularidad. En Sep-Oct.04 no se hallaron correlaciones estadísticas, lo que sugiere otros factores, por ejemplo concentración por algún proceso de advección.

López et al., (2005) también consideraron que en el OPC la oferta alimentaria puede favorecer la proliferación de diversos zoopláncteres, incluidas las hidromedusas, pues favorecen la reproducción y el mantenimiento de esos organismos gelatinosos (Suárez et al., 1999, 2000, Canché y Catellanos 2005). En los puntos de bajas densidades, podría ocurrir lo contrario, i. e., impacto de depredadores (Mills 1993, Purcell et al., 2000, Mianzan et al., 2001, Purcell y Arai 2001). No se hallaron registros de hidromedusas en contenidos estomacales de peces en el OPC.

Sólo en Jun-Jul.01 se obtuvieron correlaciones significativas (Spearman) entre la biomasa de mesozooplancton y la temperatura $(-0,460)$, y entre ésta y la densidad de hidromedusas $(-0,391)$. Con respecto a esto, en la costa central del Pacífico mejicano, entre 1995 y 1998 Franco et al., (2004) observaron coincidencia entre las mayores biomasas de zooplancton y las menores temperaturas superficiales, incluso durante El Niño 97-98, mostrando el impacto de la temperatura sobre esa comunidad. Algo similar ocurrió en el Pacífico de Chile con las hidromedusas, pero a nivel específico, aunque también se observó lo contrario (Villenas et al., 2009). En Bahía Culebra (Costa Rica) la máxima abundancia promedio de hidromedusas $\left(2.1 \pm 4.3 / \mathrm{m}^{3}\right)$ coincidió con el afloramiento costero, indicado por alta concentración de oxígeno y baja temperatura. Estos ejemplos sugieren que hay cambios en la influencia de la temperatura sobre las hidromedusas, dependiendo de la región, época y composición de especies.

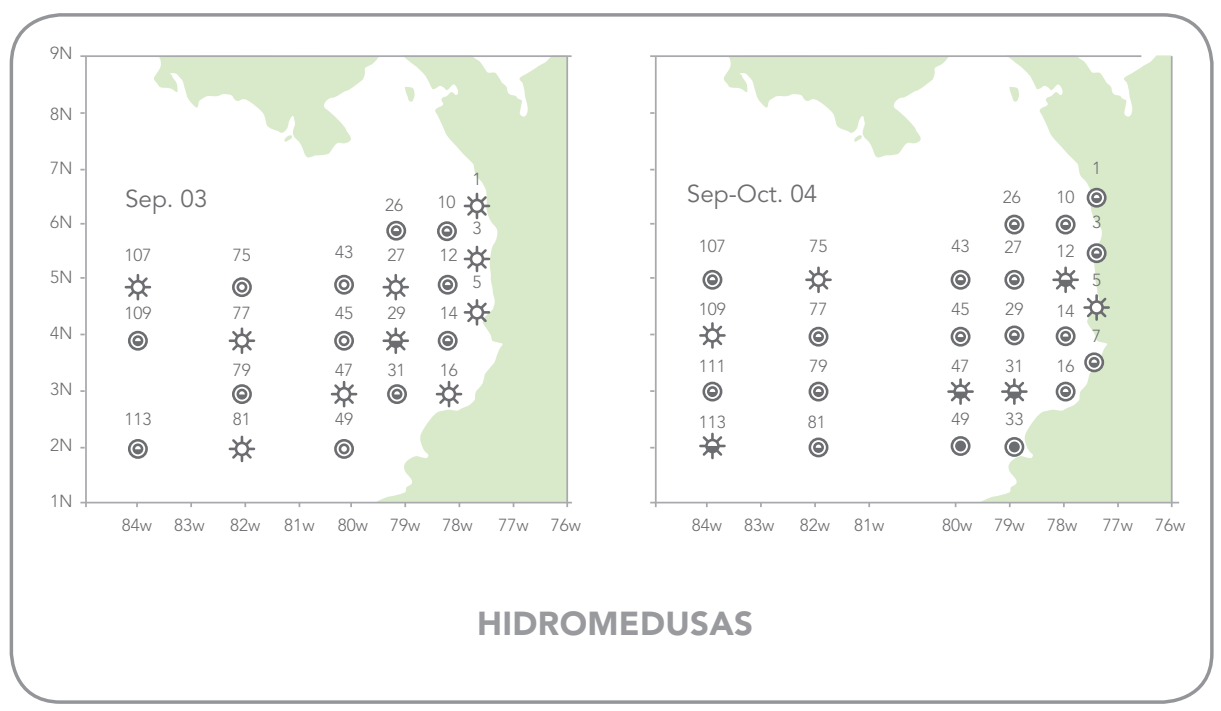

ISSN 1900-4699 • Volumen 9 • Número 1 • Páginas 108-131 • 2013 


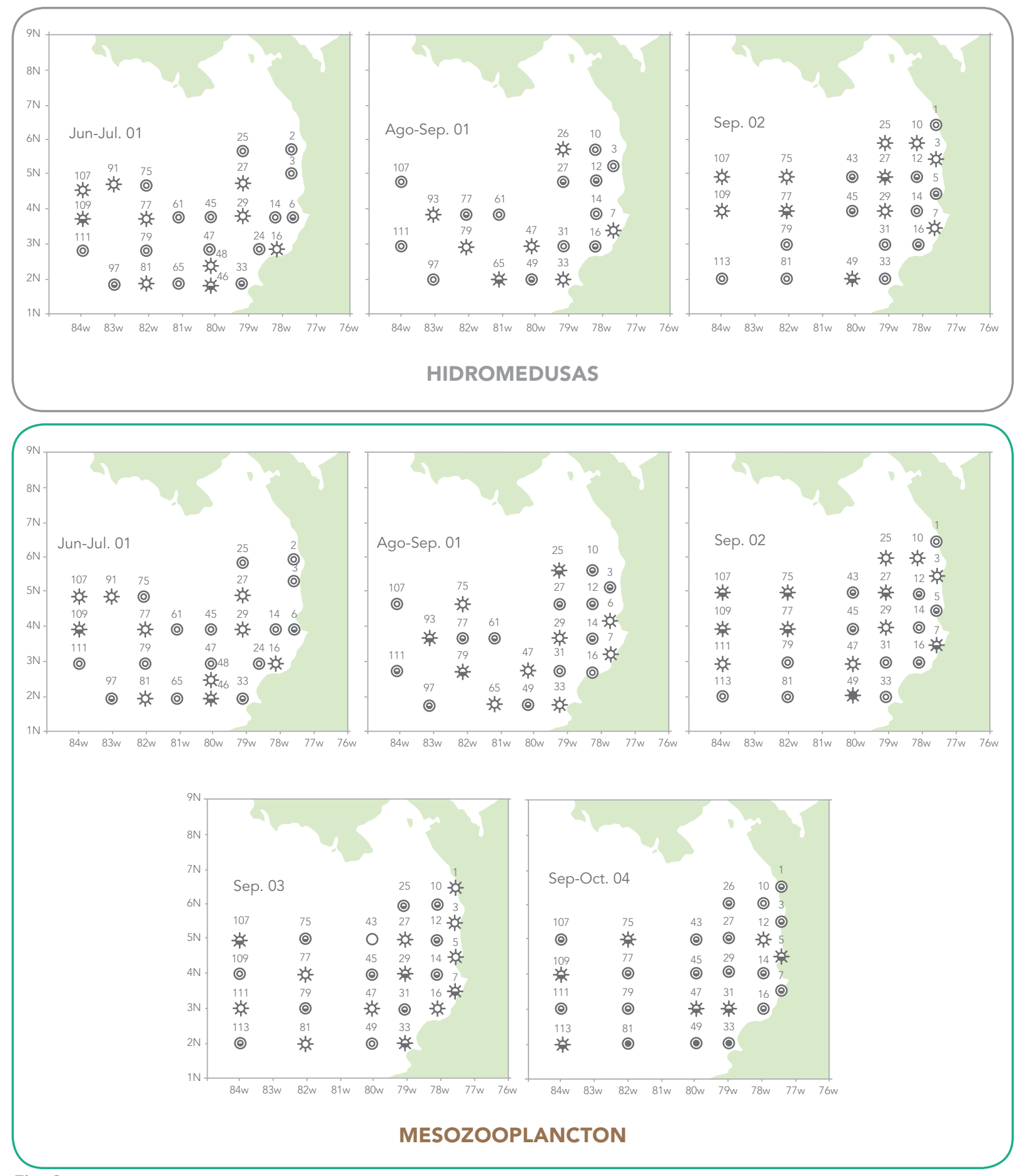

Fig. 3. Distribución de densidades totales de hidromedusas (ind/100 $\mathrm{m}^{3}$ ) y biomasas de mesozooplancton ( $\mathrm{g} / 100 \mathrm{~m}^{3}$ ) en la superficie del OPC durante los cinco periodos de estudio. Valores bajos o medios - altos O: Hidromedusas <16 16-205 >205; biomasas mesozooplancton <2,42 2,42 $19,00>19,00$. Estaciones diurnas Estaciones nocturnas $\mathrm{O}$ 
La abundancia, distribución y diversidad del zooplancton gelatinoso son influenciadas fuertemente por la salinidad y la temperatura. En sectores neríticos expuestos a la descarga de aguas continentales son pocas las especies de hidromedusas que tienen un rango de tolerancia más amplio (Palma y Rosales 1995, Benović et al., 2000, Suárez et al., 2002, Palma y Apablaza 2004). Así mismo, las salinidades altas impactan negativamente sobre la densidad de buen número de especies de hidromedusas, contribuyendo a marcar límites en su distribución nerítica (Canché y Castellanos 2005). En el OPC se observó algo similar, pues las mayores densidades de hidromedusas tendieron a observarse en aguas intermedias y costeras.

Cely y Chiquillo (1993) las encontraron a lo largo de la costa, con menor presencia en aguas litorales; según su argumento, porque la temperatura y salinidad fueron inferiores al rango de tolerancia de las especies halladas. López (1997) observó densidades más bajas en septiembre que en febrero-marzo y concentraciones mayores hacia el norte, y algunos focos en las áreas centro y sur de la zona nerítica, imputándolas al afloramiento norteño y al efecto permanente de las células de circulación ciclónica en dichas áreas (Alvariño 1978).

Es oportuno recordar que, sobre todo, son los patrones de circulación los que definen la distribución del zoopancton en general (Harris et al., 2000, Flynna y Fashamb 2002, Sutor 2005). En el OPC apenas se ha esbozado un régimen topográfico superficial general (CCCP, 2002), que menciona corrientes superficiales, frentes, remolinos, convergencias, divergencias y surgencias, no caracterizados todavía. Durante los periodos contemplados tampoco se estudió esta dinámica, de forma que no fue posible analizar este aspecto.

Estructura taxonómica y distribución de las hidromedusas

Se obtuvieron 4628 ejemplares de 18 especies, nueve meroplanctónicas y nueve holoplanctónicas, de los órdenes Anthoathecata, Leptothecata, Narcomedusae y Trachymedusae (Tabla 2). Aun así, este número es importante, ya que para el OPC se han identificado 27 géneros y 40 especies (Baldrich y López 2010).

Aglaura hemistoma, Lyripe tetraphylla y Cytaeis tetrastyla constituyeron la mayor proporción (\%), con 60,$18 ; 20,24$ y 9,64, respectivamente; su frecuencia espacial también tuvo este orden (López y Baldrich 2010). En orden de abundancia siguieron SolmundeIla bitentaculata (2,96\%) y Sarsia coccometra (2,85\%); los demás taxa tuvieron abundancias menores. Las tres primeras especies son resistentes a los cambios térmicos y halinos, lo que ha facilitado su distribución cosmopolita, incluso en el Pacífico americano (Segura 1984). Esto contrasta con la escasa representatividad de A. hemistoma y C. tetrastyla reportada por Alvariño (1978) y Cely y Chiquillo (1993) para aguas costeras del OPC. Alvariño (1999) y Suárez et al., (1999) indican que es normal que las poblaciones planctónicas de cnidarios estén dominadas por pocas especies habituales y ampliamente distribuidas,

Las mayores concentraciones en algunos sectores se relacionarían, en parte, con la disponibilidad de alimento, pues las hidromedusas incluyen en su dieta un amplio espectro de zooplánctere. 
lo cual es una de las características de las comunidades de hidromedusas, en general.

Hay algunas similitudes con aguas de Costa Rica (Rodríguez et al., 2012) y Chile (Segura 1984, Palma y Rosales 1995, Apablaza y Palma 2003, 2006, Palma y Apablaza 2004, Palma et al., 2007, Pavez et al., 2010, Bravo et al., 2011), en cuanto a que Aglaura hemistoma, Lyriope tetraphylla, Ropalonema velatum, Solmundella bitentaculata, Sarsia coccometra y Clytia spp., son comunes y dominan la comunidad de hidromedusas en diferente grado. Algo parecido ocurre en México, donde además de estas especies, pueden predominar Cytaeis tetrastyla y Pegantha triloba (Segura et al., 2003, 2010), además de que se ha reportado $S$. coccometra por primera vez (Segura et al., 2010). Las similitudes señaladas quizás corresponden a que dichas especies pertenecen a la misma provincia biogeográfica, pero hacen falta estudios en el OPC que incluyan otros meses de año para aseverarlo.

Para las bahías de Panamá y Santa Helena (Ecuador) no se han reportado las especies presentes en el OPC, excepto Clytia spp., en el primer caso (Miglietta et al., 2008) y Solmundella bitentaculata en el segundo (Andrade 2012).

Debido al estado de deterioro de los especímenes de Aequorea, Clytia, Cunina, Eirene y Pegantha, no pudieron ser identificados a nivel de especie.

Los resultados reflejaron la presencia continua y la distribución heterogénea de las hidromedusas meroplanctónicas en el OPC, así como la mayor densidad y aparición en áreas más extensas de las holoplanctónicas, especialmente lejos de la costa (Ramírez y Zamponi 1981, Alvariño 1999). El bajo número o ausencia de especies meroplanctónicas no implica la ausencia en el medio, pues pueden estar presentes en sus formas bentónicas o latentes de resistencia ocultas en el sedimento (Boero et al., 1996). Una posible explicación para las proliferaciones de hidromedusas holoplanctónicas se basa en un "ajuste de la historia de vida", con base en una población remanente de pocos individuos que subsisten en algunos lugares, en condiciones adversas. Con esta congregación maximizan la dispersión de gametos y se reproducen cuando las condiciones sean favorables, produciendo un número alto de individuos (CIESM 2001).

Es preciso aumentar la información sobre la ecología de las fases planctónicas y sésiles de los zooplánteres gelatinosos, si se quieren comprender las causas de sus proliferaciones masivas en ambientes perturbados o eutróficos, como muchos estuarios y ecosistemas costeros, tarea que se dificulta por el exiguo conocimiento sobre la ecología de los pólipos. En las últimas décadas se ha gestado un cambio en los océanos y en ciertas regiones los efectos acumulativos de la creciente influencia humana se han relacionado con esas propagaciones (Arai 2001, Mills 2001). Al respecto, Condon et al., (2012) concluyeron que el paradigma actual del aumento global de zooplancton gelatinoso, relacionado con el impacto antrópico, no está justificado y más bien debe desarrollarse una estrategia para abordar los cambios críticos en el mar y su efecto sobre estos organismos.

Al comparar las 16 estaciones comunes entre los cinco periodos, con base en las densidades de las hidromedusas, los valores de agrupación fueron relativamente altos, ya que los grupos se conformaron a niveles de similaridad entre 50 y $80 \%$. En los grupos $A, B$ y $C$ se reunieron estaciones costeras, intermedias y oceánicas, por las especies dominantes Aglaura hemistoma, Cytaeis tetrastyla y Lyriope tetraphylla; en D dos intermedias, por A. hemistoma y P. triloba; en E una costera y otra intermedia también por las tres especies dominantes, además de Sarsia coccometra, Porpita porpita y Pegantha triloba; en F costeras e intermedias, por C. tetrastyla y $S$. coccometra. La estación 75 apareció aislada, con A. hemistoma y L. tetraphylla. El análisis de ordenación mostró resultados análogos a los obtenidos en el de clasificación (Fig. 4). 

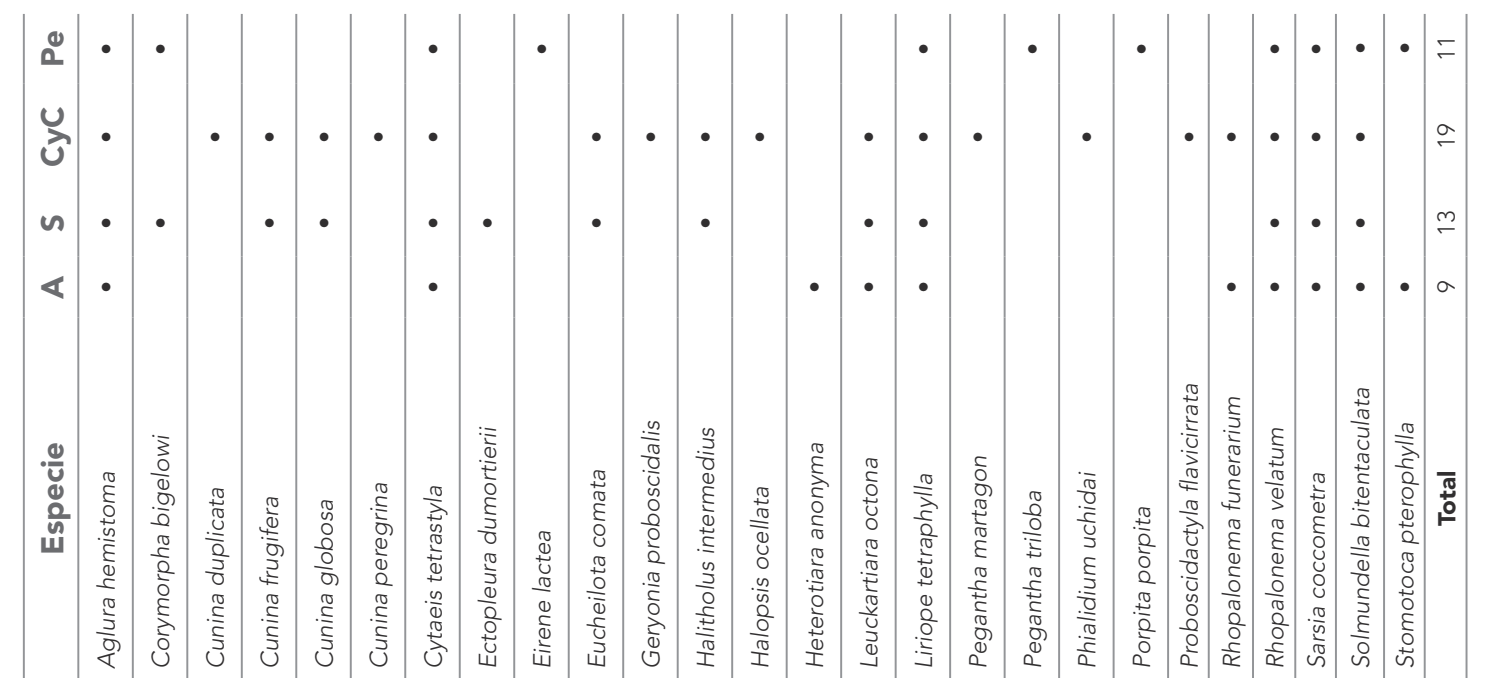

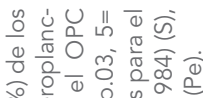

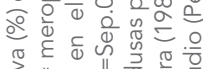

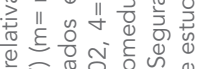

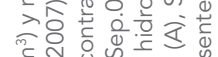

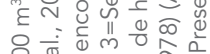

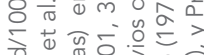

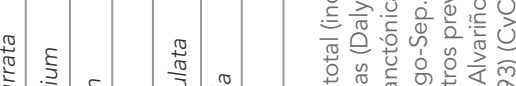

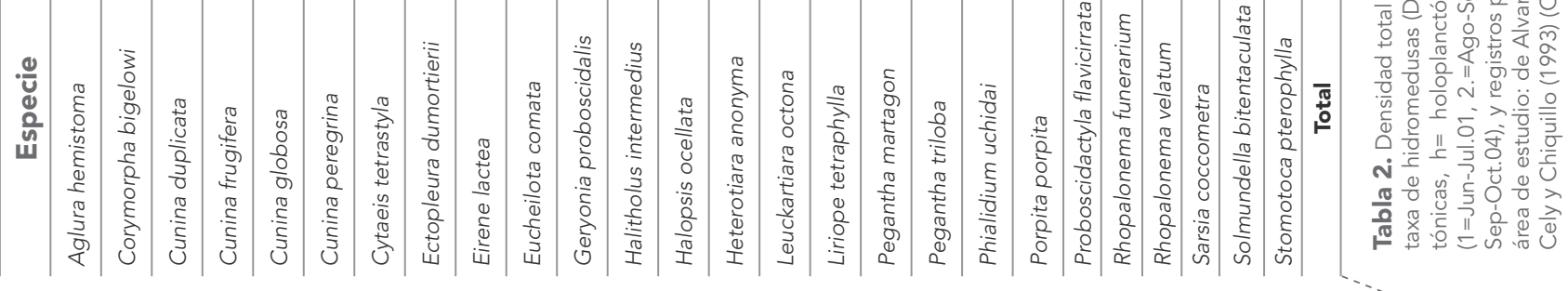

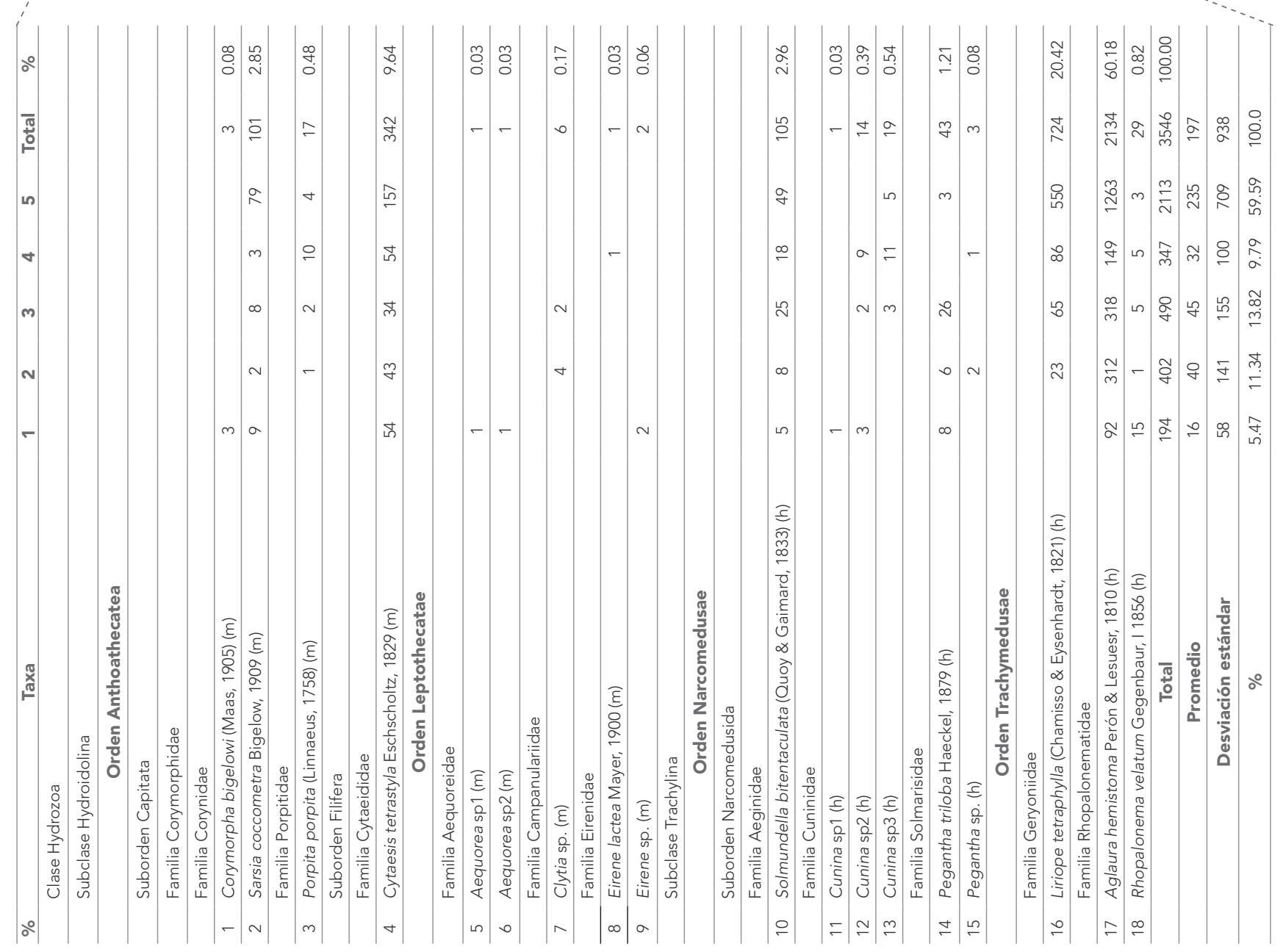


Cabe resaltar el papel de las tres especies dominantes, por ser cosmopolitas, de amplia distribución y euritípicas en el Pacífico oriental (Segura, 1984, Alvariño 1999, Suárez et al., 1999). En la plataforma del Pacífico mejicano sobrevinieron las especies neríticooceánicas y las plenamente oceánicas: en su parte externa Aglaura hemistoma y Solmundella bitentaculata, con mayor abundancia y en la interna $A$. hemistoma y Lyriope tetraphylla. La distribución amplia de las dos primeras especies y los procesos advectivos desde la zona oceánica ocultaron un gradiente definido en aguas neríticas (Segura et al., 2010).

Del análisis inverso de Kaandorp se obtuvieron los grupos de especies exclusivas (Aequorea sp. 1, Aequorea sp. 2, Eirene lactea, Eirene sp. y Pegantha sp.) y características (Cunina sp. 2 y Pegantha triloba), mientras las demás fueron generalistas (Tabla 3). Se estima que esta discontinuidad en la tipología de especies en los cinco periodos avaluados refleja la complejidad oceanográfica del OPC y se hace hincapié en que, además de las variables abióticas y bióticas consideradas, es muy probable que otras influencien la comunidad de hidromedusas; en especial, la dinámica hidrológica, que incluye corrientes superficiales, frentes, remolinos, convergencias, divergencias y surgencias, comunes en el OPC (CCCP, 2002), y que -como es bien conocido- definen en gran medida la distribución del plancton en general (Harris et al., 2000, Flynna y Fashamb 2002, Sutor 2005).

Los valores del predominio y la uniformidad fueron explicados en gran parte por la densidad y frecuencia de las especies dominantes (A. hemistoma, L. tetraphylla y C. tetrastyla), si se tiene en cuenta que la distribución uniforme del plancton gelatinoso está relacionada con su alta adaptabilidad (Suárez et al., 1999), como es el caso de estas especies (Segura 1984, Alvariño 1999, Suárez et al., 1999).

Es importante conocer la biodiversidad planctónica pues hay cambios en la composición de especies que pueden afectar los flujos de materia y energía, así como las interacciones tróficas que favorecen la abundancia de las especies que controlan estos flujos (Chapin et al., 2000). De acuerdo con Margalef (1961, 1986), el cálculo de la diversidad en las poblaciones zooplanctónicas basado en uno o pocos grupos y no en la totalidad de las poblaciones, varía entre 3,50 y 4,50.

Los cinco periodos de estudio se caracterizaron por la baja riqueza y diversidad de hidromedusas; no obstante la menor densidad total y promedio (293 y 16 ind/100 $\mathrm{m}^{3}$ ), los valores más representativos $\left(S=13, H^{\prime}=1,65\right.$ bits) se registraron en JunJul.01. En este tipo de información hay diferencias, a veces muy marcadas, dependiendo de la región del Pacífico, época (lluviosa o seca, surgencia),

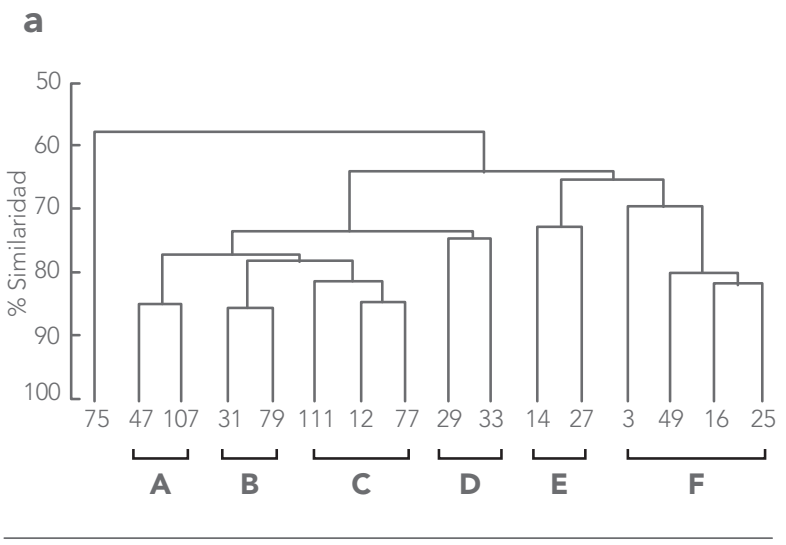

b

75
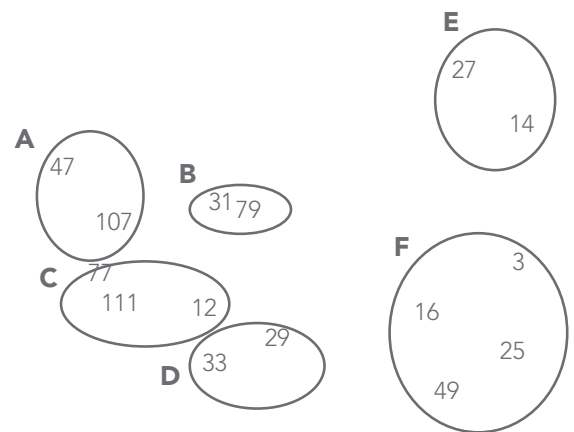

Figura 4. a. Dendrograma de clasificación (índice cofenético $=0.84$ ) y b. NMDS (estrés $=0,14$ ) de las 16 estaciones comunes entre los cinco periodos de muestreo en el OPC, elaborados con las densidades de hidromedusas. 
transporte de especies por las corrientes y aportes de origen terrestre (Tabla 4).

En el OPC no es posible realizar este tipo de confrontaciones, pues los cruceros ocenográficos del ERFEN se realizan sólo hacia septiembre, dentro de la época cálida, generalmente lluviosa (Rodríguez et al.,2003, Devis et al., 2008).

\section{Variación nictimeral de las hidromedusas}

La distribución de las hidromedusas también está relacionada con los desplazamientos nictimerales de un alto porcentaje de especies, provocados por factores físicos y biológicos, como la intensidad de la luz, mezcla vertical, alimentación, escape a la depredación y ganancia energética, comportamiento demostrado igualmente en otros grupos gelatinosos, como los quetognatos y sifonóforos (Alvariño 1999, Vinogradov y Shushkina 2002, Benović et al., 2005, Apablaza y Palma 2006).

En el OPC las mayores densidades noche vs. día (ind/100 $\mathrm{m}^{3}$ ) en Ago-Sep.01 (26,58 vs. 5,78), Sep.02 $(26,19$ vs. 13,23), Sep.03 (23,17 vs. 4,80) y Sep-Oct.04 $(108,09$ vs. 35,70$)$ (Fig. 5), serían un indicativo de tales desplazamientos a la superficie durante la noche, como se ha comprobado, por ejemplo en Chile (Apablaza y Palma 2006, Bravo et al., 2011). Con poca diferencia, lo contrario acaeció en Jun-Jul.01 (6,31 vs. $12,63)$ y se explicaría porque algunas hidromedusas tienen migraciones inversas y otras no lo hacen ( $\mathrm{Vi}$ nogradov y Shushkina 2002, Benović et al., 2005). La correlación de Spearman reflejó lo expuesto, sólo en Sep.03 $(0,618)$ y Sep-Oct.04 (0,527).

El alimento de las hidromedusas, en este caso representado por las biomasas de mesozooplancton, del mismo modo evidenció los valores más altos en la noche, seguramente por sus migraciones verticales a la superficie, como lo sustentan varios autores (e. g., Sthephen y Forst 1992, Tattsuki y Yamamura 1999, Franco et al., 2004, Färber et al., 2006). Este sería el fundamento de la correlación entre entre tales biomasas y la variable día:noche para Ago-Sep.01 (Spearman= 0,489). En todo caso, los desplazamientos circadianos de las hidromedusas en el OPC deben ser estudiados de manera profunda, considerando cada especie, lo cual no se efectuó en este trabajo, por falta de información particular.

En el análisis general de las características estructurales de la comunidad de hidromedusas en el OPC emprendido en este trabajo, es importante recalcar que los trabajos consultados, la mayoría de otras latitudes de América, pues en el OPC son escasos, se han realizado en diferentes periodos, con metodologías, y frecuencias y áreas de muestreo disímiles. Además, las campañas oceanográficas del ERFEN en Colombia, por lo general sólo cubren apenas ca. 25 días y se efectúan dentro

\begin{tabular}{|c|c|c|c|c|c|c|c|c|c|c|c|c|}
\hline Índice & $\begin{array}{l}\text { Jun- } \\
\text { Jul.01 }\end{array}$ & $\begin{array}{c}\text { Ago- } \\
\text { Sep.01 }\end{array}$ & $\begin{array}{c}\text { Sep. } \\
02\end{array}$ & $\begin{array}{c}\text { Sep. } \\
03\end{array}$ & $\begin{array}{c}\text { Sep. } \\
04\end{array}$ & a & b & c & d & e & $f$ & g \\
\hline$H^{\prime}$ & 1,65 & 0,87 & 1,25 & 1,59 & 1,11 & $>1,46$ & & & $>2,50$ & $\begin{array}{l}0,29- \\
3,03\end{array}$ & $\begin{array}{l}2,68- \\
7,40\end{array}$ & $\begin{array}{l}0,20- \\
1,50\end{array}$ \\
\hline$J^{\prime}$ & 0,64 & 0,38 & 0,52 & 0,50 & 0,50 & & & & & & $\begin{array}{l}0,88- \\
0,91\end{array}$ & $\begin{array}{l}0,10- \\
0,85\end{array}$ \\
\hline 1-Lambda & 0,75 & 0,38 & 0,55 & 0,57 & 0,57 & & & & & & & \\
\hline S & 13 & 10 & 11 & 11 & 9 & 22 & 23 & 17 & & & & $\begin{array}{l}0,28- \\
1,40\end{array}$ \\
\hline
\end{tabular}

Tabla 4. Diversidad de Shannon $\left(H^{\prime}\right)$, uniformidad de Pielou $\left(J^{\prime}\right)$, predominio de Simpson (1-Lambda) y riqueza de especies $(S)$ de hidromedusas por periodo de estudio, y algunos valores comparativos. a= Zona costera OPC (Cely y Chiquillo (1993). b-e= Chile (Apablaza y Palma 2003, Palma y Apablaza 2004, Palma et al., 2007, Villenas et al., 2009) f= Ecuador (Andrade 2012, Ruiz 2010). g= México (Segura et al.,2009). 


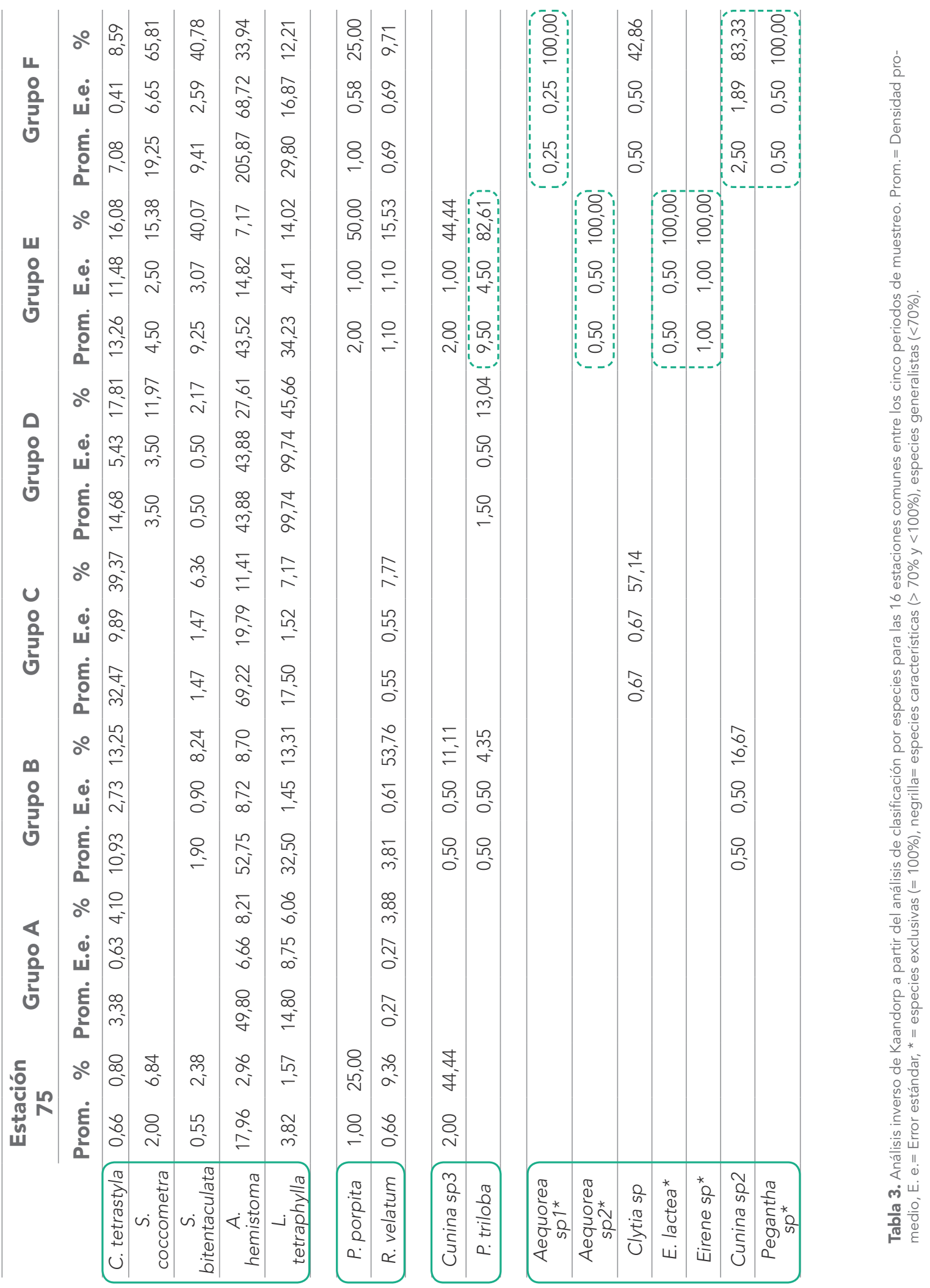


de la época hidrográfica cálida (mayo-diciembre), definida por la ubicación $\approx 10^{\circ} \mathrm{N}$ de la ZCIT (Rodríguez et al., 2003, Devis et al., 2008), casi exclusivamente hacia los meses de septiembre (a veces en febrero-marzo), lo cual hace más difícil la interpretación de los datos. Lo anterior no da margen para mayores comparaciones.

\section{CONCLUSIONES}

La temperatura y salinidad superficiales en los cinco periodos estudiados estuvieron dentro de los rangos pre-establecidos para el OPC; espacialmente manifestaron el patrón general habitual, caracterizado por un gradiente térmico que disminuye de las aguas costeras hacia las oceánicas, mientras que con la salinidad ocurre lo contrario.

La comunidad de hidromedusas no presentó un patrón de distribución espacial definido en ninguno de los aspectos analizados (densidad, composición e índices ecológicos). Se cree que esto responde a la conocida distribución general heterogénea del zooplancton debida a múltiples factores, e. g., corrientes, temperatura, salinidad, disponibilidad de alimento, y migraciones circadianas, principalmente. Las mayores densidades de hidromedusas en Sep.02 y Sep-Oct.04, posiblemente se debieron a mejores condiciones generales que favorecieron su desarrollo.
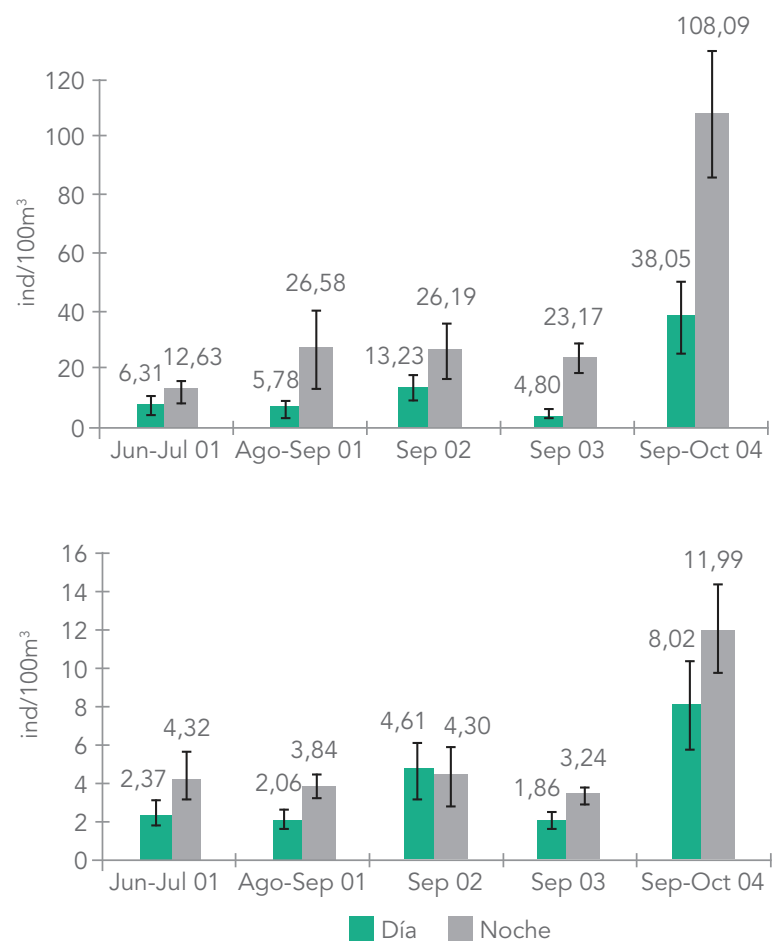

Fig. 5. Promedios día:noche de la densidad de hidromedusas (ind/100 $\mathrm{m}^{3}$ ) y la biomasa de mesozooplancton $\left(\mathrm{g} / 100 \mathrm{~m}^{3}\right)$ en la superficie del OPC durante los cinco periodos de estudio. Se indican los valores promedio y las barras de error estándar.

Se destacaron A. hemistoma, L. tetraphylla y C. tetrastyla por su predominancia a lo largo del estudio, considerándose, por lo tanto, como euritípicas del OPC. Que no se haya observado una secuencia clara en la aparición de especies

\section{A. emistoma, L. tetraphylla y C. tetrastyla se consideran euritípicas del OPC.}


exclusivas, características o generalistas, y que la similaridad entre las estaciones se haya explicado por los valores de las variables abióticas o bióticas consideradas, más que por su ubicación (costeras, intermedias u oceánicas) o por la hora de los registros, refleja la complejidad oceanográfica del OPC; la poca utilidad de los análisis estadísticos contribuyeron a fundamentarlo. También hay que considerar que hay especies mero- y holoplanctónicas, cuyos ciclos de vida son aún desconocidos o poco comprendidos.

Hay indicios de que las diferencias entre los promedios de densidad de hidromedusas día: noche se deban, entre otras causas, a la migración nocturna que muchas especies realizan hacia la superficie, por las diferentes razones ya expuestas.

\section{AGRADECIMIENTOS}

Esta investigación fue financiada y ejecutada por la Universidad Militar Nueva Granda, con el soporte de la Dirección General Marítima (DIMAR) -consistente en permitir la participación en los cruceros oceanográficos del ERFEN- y de su Centro Control de Contaminación del Pacífico (CCCP), que registró y suministró los datos oceanográficos. Los autores expresan sus sentimientos de reconocimiento a estas entidades y a las especialistas en la taxonomía de hidromedusas, María de Lourdes Segura Puertas (D. E. P.) y Laura Celis, del Laboratorio de Plancton del Instituto de Ciencias del Mar y Limnología de la Universidad Nacional Autónoma de México, Unidad Académica Puerto Morelos, quienes confirmaron las identificaciones. 


\section{BIBLIOGRAFÍA}

1. Alvariño A. 1978. El zooplancton del Pacífico colombiano y las Pesquerías, p. 206-271. En Vegas M y Rojas R. (Eds). Memorias I Seminario de Oceanografía del Pacífico Sudamericano, Cali (septiembre 1-5/76).

2. Alvariño A. 1985. Predation in the plankton realm; mainly with reference to fish larva. Investigaciones Marinas CIMAR, 2(1): 1-122.

3. Alvariño A. 1999. Hidromedusas: abundancia batimétrica diurna-nocturna y estacional en aguas de California y Baja California, y estudio de las especies en el Pacífico oriental y otras regiones. Revista de Biología Marina y Oceanografía, 34(1): 1-90.

4. Andrade C. 2012. Distribución estacional y ecología de las medusas (Cnidarias: Hydrozoa) en la zona costera sur de la bahía de Santa Elena durante el período (octubre 2004-octubre 2005). Acta Oceanográfica del Pacífico, 17(1): 127-138.

5. Apablaza P y Palma S. 2003. Hidromedusas de los canales australes colectadas entre la Boca del Guafo y Canal Pulluche, p. 139-145. En: Libro de Resúmenes: Taller sobre los resultados del crucero CIMAR 8 Fiordos, Valparaíso,

6. Apablaza P y Palma S. 2006. Efecto de la zona de mínimo oxígeno sobre la migración vertical de zooplancton gelatinoso en la bahía de Mejillones. Investigaciones Marinas Valparaíso, 34: 81-95.

7. Arai MN. 2001. Pelagic coelenterates and eutrophication: a review. Hydrobiologia, 451(155): 69-87.
8. Baldrich AM y López RH. 2010. Hidromedusas mesozooplanctónicas del océano Pacífico colombiano. Biota Colombiana, 11(1-2): 3-11.

9. Benović A, Lučić D y Onofori V. 2000. Does change in an Adriatic hydromedusan fauna indicate an early phase of marine ecosystem destruction? Marine Ecology, 21 (3-4): 221-231.

10. Benović $A$, Lučić $D$, Onofori $\vee$, Batistić M y Njire J. 2005. Bathymetric distribution of medusae in the open waters of the middle and south Adriatic Sea during spring 2002. Journal of Plankton Research, 27(1): 79-89.

11. Bi H, Peterson WT y Strub PT. 2011. Transport and coastal zooplankton communities in the northern California Current system. Geophysical Research Letters, 38: 1-5.

12. Bouillon J. 1999. Hydromedusae, p. 385-465. En: Boltovskoy D. (Ed.). South Atlantic Zooplankton, Vol I. Backhuys Publishers, Leiden.

13. Bouillon J y Boero F. 2000. The Hydrozoa: A new classification in the ligth of old knowledge. Thalassia Salentina, 24: 3-45.

14. Bravo V, Palma S y Silva N. 2011. Seasonal and vertical distribution of medusae in Aysén region, southern Chile. Latin American Journal of Aquatic Research, 39(2): 359-377.

15. Canché E y Castellanos I. 2005. Medusas (Cnidaria) de la bahía de la Ascensión Quintana Roo. Hidrobiológica, 1(15): 65-72.

16. Cely H y Chiquillo J. 1993. Quetognatos, sifonóforos e hidromedusas de la región costera del Pacífico colombiano. Trabajo de Grado Facultad de Biología Marina, Universidad Jorge Tadeo Lozano, Santa Fe de Bogotá, 120 p. 
17. CCCP. 2002. Compilación oceanográfica de la Cuenca Pacífica colombiana. Centro Control de Contaminación del Pacífico, Tumaco, 109 p.

18. Chapin FS III, Zavaleta ES, Eviner VT, Naylor RL, Vitousek PM, Reynolds HL, Hooper DU, Lavorel S, Sala OE, Hobbie SE, Mack MC y Díaz S. 2000. Consequences of changing biotic diversity. Nature 405: 234-242.

19. Chávez FP, Bertrand A, Guevara R, Soler P y Csirke J. 2008. El sistema del norte de la Corriente de Humboldt, historia breve, estatus actual y una vista hacia el futuro. Progress in Oceanography, 79(2-4): 3-15.

20. CIESM. 2001. Gelatinous zooplankton outbreaks: theory and practice. The Mediterranean Science Commission (CIESM), Nápoles, 29.Ago01.Sep01. Workshop Monographs, 14:1-112.

21. Daly M, Brugler MR, Cartwright P, Collins A G, Dawson MN, Fautin DG, France SC, Mcfadden CS, Opresko DM, Rodriguez E, Romano SL y Stake JL. 2007. The phylum Cnidaria: A review of phylogenetic patterns and diversity 300 years after Linnaeus. Zootaxa, 1668: 127-182.

22. Devis A. 2003. Evolución del evento El Niño 2002-2003 y efectos sobre la cuenca del Pacífico colombiano y la bahía de Tumaco. Boletín Científico CCCP, 10: 15-30.

23. Fernández A y Färber J. 2006. Zooplancton and the oceanography of the eastern tropical Pacific: a review. Progress in Oceanography, 69: 318-359.

24. Flynna KJ y Fashamb MJR. 2002. A modelling exploration of vertical migration by phytoplankton. Journal of Theoretical Biology, 218(4): 471-484.
25. Forsbergh E. 1963. Algunas características de las variables meteorológicas, hidrográficas y biológicas en el Golfo de Panamá. Comiisón Interamericana del Atún Tropical, 7(1): 55-91.

26. Franco C, Godínez E, Filonov A, Tereshchenko E y Freire J. 2004. Plankton biomass and larval fish abundance prior to and during the El Niño period of 1997-1998 along the central Pacific coast of Mexico. Progress in Oceanography, 63: p. 99-23.

27. Boero F, Belmonte G, Fanelli G, Piraino S, Rubino F. 1996. The continuity of living matter and the discontinuities of its constituents: do plankton and benthos really exist? Trends in Ecology and Evolution, 11: 177-180.

28. Condon RH, Graham WM, Duarte CM, Pitt KA, Lucas CH, Haddock SHD, Sutherland KR, Robinson $\mathrm{KL}$, Dawson MN, Decker MB, Mills CE, Purcell JE, Malej A, Mianzan H, Uye S, Gelcich S y Madin LP. 2012. Questioning the Rise of Gelatinous Zooplankton in the World's Oceans. Bioscience, 62(2): 160-169.

29. Graham WM, Pagès F, Hammer WM. 2001. A physical context for gelatinous zooplankton aggregations: a review. Developments in $\mathrm{Hi}$ drobiology, 155: 199-212.

30. Hair J, Anderson R, Tatham W y Black, W. 1999. Análisis multivariante. Prentice Hall Iberia, Madrid, $91 \mathrm{p}$.

31. Hansson LJ, Moeslund $O$, Kiørboe Ty Riisgård HU. 2005. Clearance rates of jellyfish and their potential predation impact on zooplankton and fish larvae in a neritic ecosystem (Limfjorden, Denmark). Marine Ecology Progress Series, 304: 117-131. 
32. Hansson LJ y Kiørboe T. 2006. Prey-specific encounter rates and handling efficiencies of prey selectivity in ambush-feeding hydromedusae. Limnology and Oceanography, 51: 1849-1858.

33. Harris RP, Wiebe P, Lenz J, Skjoldal H y Huntley M. 2000. ICES Zooplankton methodology manual. Academic Press, Salt Lake City, 538 p.

34. Jiménez IL. 2008. Prospección del ictioplancton en la superficie del océano Pacífico colombiano. Periodo 19.Sep-08.Oct.04. Trabajo de Grado Programa Biología Aplicada, Facultad de Ciencias Básicas, Universidad Militar Nueva Granada, Bogotá D. C., 115 p.

35. Johnson W S y Allen DM. 2005. Zooplankton of the Atlantic and Gulf coasts: a guide to their identification and ecology. Johns Hopkins University Press, Baltimore, $379 \mathrm{p}$.

36. Kaandorp JA 1986. Rocky substrate communities of the infralittoral fringe of the Boulonnais coast, NW France: a quantitative survey. Marine Biology, 92: 255-265.

37. Kramp PL. 1961. Synopsis of the medusae of the world. Journal of the Marine Biological Association of the United Kingdom, 40: 1-469.

38. López RH. 1997. Das Fischplankton der pazifischen Küstengewaesser Kolumbiens. Reihe Biologie, Tectum, Marburg, 93: 1-522.

39. López RH, Uribe J, Escobar N, López C y Riveros D. 2005. Mesozooplancton superficial del Océano Pacífico Colombiano durante los cruceros de 2001, 2002 y 2003 de la serie ERFEN (Estudio Regional del Fenómeno El Niño). Informe final Proyecto CIAS-2003-003. Programa.
Biología Aplicada, Facultad de Ciencias Básicas, Universidad Militar Nueva Granada, Bogotá D. C., 74 p.

40. López RH y Baldrich ÁM. 2010. Hidromedusas del Océano Pacífico Colombiano. Catálogo básico de identificación rápida I. Ed. Panamericana Bogotá, $79 \mathrm{p}$.

41. Luzuriaga de CruzM.2009. Distribución espaciotemporal del ictioplancton y zooplancton marino en las islas Galápagos, Ecuador (1988-2001). Acta Oceanográfica del Pacifico, 15(1): 97-119.

42. Mackas DL y Galbraith MD. 2002. Zooplankton distribution and dynamics in a North Pacific eddy of coastal origin: I. Transport and loss of continental margin species. Journal of Oceanography, 58: 725-738.

43. Margalef R. 1958. Information theory in ecology. General Systems, 3: 36-71.

44. Margalef, R. 1961 Communication of structure in planktonic populations. Limnology and Oceanography, 6:124-128.

45. Margalef R. 1986. Ecología. Ediciones Omega S. A., Barcelona, $951 \mathrm{p}$.

46. Mattos H y Mujica A. 2012. Composición de zooplancton superficial en zonas lisas y rugosas en la bahía de Coquimbo (noviembre 2001). Latin American Journal of Aquatic Resarch, 40(2): 453-461.

47. Mayer AG. 1910. Medusae of the world: The Hydromedusae. Volume I. Carnegie institution of Washington, Washington, D.C., 238 p.

48. Mianzan H, Pájaro M, Álvarez G y Madirolas A. 2001. Feeding on survival-food: gelatinous 
plankton as a source of food for anchovies. Developments in Ecology, 155: 45-53.

49. Miglietta MP, Rossi M y Collin R. 2008. Hydromedusa blooms and upwelling events in the Bay of Panama, Tropical East Pacific. Journal of Plankton Research, 30(7): 783-793.

50. Mills CE. 1993. Natural Mortality in NE Pacific Coastal Hydromedusae: Grazing Predation, Wound Healing and Senescence. Bulletin of Marine Science, 53(1): 194-203.

51. Mills CE. 2001. Jellyfish blooms: Are populations increasing globally in response to changing ocean conditions? Hydrobiologia, 451: 55-68.

52. Morales A y Brugnoli E. 2001. El Niño 19971998 impact on the plankton dynamics in the Gulf of Nicoya, Pacific coast of Costa Rica. Revista de Biología Tropical, 49(Supl. 2): 103-114.

53. Morales A. 2008. Caracterización cualitativa del zooplancton del Área de Conservación Marina Isla del Coco (ACMIC), Océano Pacífico de Costa Rica. Revista de Biología Tropical, 56 (Supl. 2): 159-169.

54. Møller LF y Riisgård HU. 2007. Feeding, bioenergetics and growth in the common jellyfish, Aurelia aurita, and two hydromedusae (Sarsia tubulosa and Aequorea vitrina). Marine Ecology Progress Series, 346: 153-165.

55. Pagès F, Gili J. y Bouillon J. 1992. Medusae (Hidrozoa, Scyphozoa, Cubozoa) of the Benguela current (Southeastern Atlantic). Scentia Marina, 56(1):1-64.

56. Palma S y Apablaza P. 2004. Abundancia estacional y distribución vertical del zooplancton gelatinoso carnívoro en un área de surgencia en el norte del Sistema de la Corriente de Humboldt. Investigaciones Marinas Valparaíso, 32 (1): 49-70.

57. Palma S, Apablaza P y Silva N. 2007. Hydromedusae (Cnidaria) of the Chilean southern channels (from the Corcovado Gulf to the PullucheChacabuco Channels). Scientia Marina, 71(1): 65-74.

58. Palma S y Rosales S. 1995. Composición, distribución y abundancia estacional del macroplancton de la bahía de Valparaíso. Investigaciones Marinas Valparaíso, 23: 49-66.

59. Pavez MA, Landaeta MF, Castro LR y Schneider W. 2010. Distribution of carnivorous gelatinous zooplankton in the upwelling zone off central Chile (austral spring 2001) Journal Of Plankton Research, 32(7): 1051-1065. 57.

60. Pielou E. 1966. The measurement of diversity in different types of biological collections. Journal of Theoretical Biology, 13: 131-144.

61. Prairie JC, Sutherland KR, Nickols KJ y Kaltenberg AM. 2012. Biophysical interactions in the plankton: A cross-scale review. Limnology and Oceanography: Fluids and Environments, 2: 121-145.

62. Prudkovsky AA. 2013. Trophic role of ambushforaging hydromedusae in the White Sea. Marine Ecology, 34(Suppl. 1): 153-164.

63. Purcell JE, Brown ED, Stokesbury KDE, Haldorson LH y Shirley TC. 2000. Aggregations of the jellyfish Aureua labiata: abundance, distribution, association with age-0 walleye pollock, 
and behaviors promoting aggregation in Prince William Sound, Alaska, USA. Marine Ecology Progress Series, 195: 145-158.

64. Purcell JE y Arai MN. 2001. Interactions of pelagic cnidarians and ctenophores with fish: A review. Hydobiologia, 451: 27-44.

65. Ramírez A. 2005. Ecología aplicada: Diseño y análisis estadístico. Universidad Jorge Tadeo Lozano, Bogotá D. C., 325 p.

66. Ramírez A. 2006. Ecología: Métodos de muestreo y análisis de poblaciones y comunidades. Pontificia Universidad Javeriana, Bogotá D. C., $271 \mathrm{p}$.

67. Ramírez F y Zamponi O.1981. Hydromedusae, p. 443-469. En: Boltovskoy D. (Ed.). Atlas de zooplancton del Atlántico Sudoccidental y métodos de trabajo con el zooplancton marino. Publicación Especial del Instituto Nacional de Investigación y Desarrollo Pesquero (INIDEP), Mar de Plata.

68. Raskoff K, Freya A, Sommer F, Hamner W y Cross K. 2003. Collection and culture techniques for gelatinous zooplankton. Biological Bulletin, 204(1): 68-80.

69. Rodríguez E, Schneider W y Abarca del Río R. 2003. On the seasonal circulation within Panama Bight derived from satellite observations of winds, altimetry and sea surface temperature. Geophysical Research Letters, 30(7): 1410-1413.

70. Rodríguez K, Vargas JA y Segura L. 2012. Medusas (Cnidaria: Hydrozoa) de una zona de afloramiento costero, Bahía Culebra, Pacífico,
Costa Rica. Revista de Biología Tropical, 60 (4): 1731-1748.

71. Shannon C. y Weaver W. 1949. The mathematical theory of communication. University of Illinois Press, Urbana Illinois, $117 \mathrm{p}$.

72. Simpson E. 1949. Measurement of diversity. Nature, 163: 688

73. Segura L. 1984. Morfología, sistemática y zoogeografía de las medusas (Cnidaria: Hydrozoa y Scyphozoa) del Pacífico Tropical Oriental. Instituto de Ciencias del Mar y Limnología. Universidad Nacional Autónoma de Méfico, México D. F., Publ. Esp. 8: 1-314.

74. Segura L, Suárez E y Celis L. 2003. A checklist of the Medusae (Hydrozoa, Scyphozoa and Cubozoa) of Mexico. Zootaxa, 194: 1-15.

75. Segura L, Franco C, Suárez E, Gasca R y Godínez E. 2010. Summer composition and distribution of the jellyfish (Cnidaria: Medusozoa) in the shelf area off the central Mexican Pacific. Revista Mexicana de Biodiversidad, 81: 103-112.

76. Stevenson M, Guillén O y Santoro J. 1970. Marine atlas of the Pacific coastal waters of South America. Univesity of California Press, Oakland, 23 p.

77. Suárez E, Segura L y Gasca R. 1999. Medusan (Cnidaria) assemblages off the Caribbean coast of Mexico. Journal of Coastal Research. 15(1): 140-147.

78. Suárez E, Gasca R, Segura L y Biggs DC. 2002. Planktonic cnidarians in a cold-core ring in the Gulf of Mexico. Anales del Instituto de 
Biología, Universidad Nacional Autónoma de México, 73(1): 19-36.

79. Suthers IM y Rissik D. (Eds.). 2009. Plankton: A guide to their ecology and monitoring for water quality. Commonwealth Scientific and Industrial Research Organization (CSIRO) Publishing, Collingwood, 272 p.

80. Sutor M. 2005. Vertical distribution patterns of plankton and their relationship to physical factors over the continental shelf off Oregon. Oregon State University, Corvallis, 594 p.

81. Tchantsev V y Cabrera E. 1998. Algunos aspectos de investigación de la formación del régimen oceanográfico en el Pacífico colombiano. Boletín Científico CCCP, 7: 7-19.

82. Villegas N. 2002. Evolución mensual de las corrientes verticales y zonas de surgencia en la Cuenca del Pacífico Colombiano-CPC. Boletín Científico CCCP, 9: 29-36.

83. Villegas N. 2003. Estabilidad de las aguas del Pacífico Colombiano. Boletín Científico CCCP, 9: 5-14.

84. Villenas F, Soto D y Palma S. 2009. Cambios interanuales en la biomasa y biodiversidad de zooplancton gelatinoso en aguas interiores de Chiloé, sur de Chile (primaveras 2004 y 2005). Revista de Biología Marina y Oceanografía, 44(2): 309-324.

85. Vinogradov M y Shushkina E. 2002. Vertical distribution of gelatinous macroplankton in the North Pacific observed by manned submersibles Mir-1 and Mir-2. Journal of Oceanography, 58: 295-303.
86. Wirtky K. 1965. Corrientes superficiales del Océano Pacífico oriental tropical. 1965. Comisión Interamericana del Atún Tropical, 9(5): 279-304.

87. Zar JH. 2010. Biostatistical analysis. 5th Ed. Pearson Prentice-Hall, Upper Saddle River, N. Jersey, $944 \mathrm{p}$. 